\title{
Near-Field Heat Flow Between Two Quantum Oscillators
}

\author{
Gabriel Barton ${ }^{1}$
}

Received: 15 July 2016 / Accepted: 26 October 2016 / Published online: 14 November 2016

(C) The Author(s) 2016. This article is published with open access at Springerlink.com

\begin{abstract}
We calculate the exact steady-state heat flow $\mathcal{P}$ between two Ohmically damped quantum oscillators 1 and 2 , with natural frequency $\omega_{0}$, interacting through their near-field dipole-dipole potential $V$. To keep them at nominally constant temperatures $T_{1}, T_{2}$ respectively, they have to be coupled to thermostats functioning in a way one must specify explicitly unless one assumes local thermal equilibrium, which would, inadequately as a rule, restrict the calculation to leading order in $V$. Here the thermostats are modelled as stretched strings, one end attached to the oscillator, and the other to an infinitely distant device ensuring that the string carries thermal noise appropriate to $T_{1}$ or $T_{2}$ in addition to whatever motion is enforced by the oscillator. Aiming at insight rather than numerics, we focus mainly on simple approximations by powers of $T_{1}$ and $T_{2}$ for weak damping in the essentially quantum low-temperature regime where $k_{B} T_{1,2} \ll \hbar \omega_{0}$. From $\mathcal{P}$ it is easy to find the heat flux between two insulating Drude-modelled half-spaces.
\end{abstract}

Keywords Casimir heat flow $\cdot$ Nonequilibrium steady states $\cdot$ Local thermal equilibrium

\section{Introduction and Conclusions}

\subsection{Objectives}

Processes in non-equilibrium steady-state microsystems are beginning to attract attention, and we propose to study, by solving it exactly, the prototype problem of the near-field-mediated heat flow $\mathcal{P}$ between two simple-harmonic oscillators, call them 1 and 2 , maintained at different nominal temperatures $T_{1}$ or $T_{2}$. This amounts to determining the short-distance limit of $\mathcal{P}$ for the classic Casimir-Polder scenario, where the exact Maxwellian interaction is replaced by the familiar nonretarded potential $V$ between the oscillators. Apart from its intrinsic interest, the result can then serve as a relatively simple check on more ambitious

Gabriel Barton

g.barton@sussex.ac.uk

1 Department of Physics \& Astronomy, University of Sussex, Brighton BN1 9QH, England 
fully-retarded calculations for arbitrary distances comparable to the oscillator wavelength. The question is timely also because many recent calculations both of $\mathcal{P}$ and of the force between 1 and 2 proceed from the assumption of local thermal equilibrium (LTE), that the state of each body is just what it would be if the body were isolated and in true thermodynamic equilibrium at $T_{1}$ or at $T_{2}$. However, we shall see that $L T E$ fails beyond leading order in $V$; whereas it is well known that leading (here the second) order in the interaction Hamiltonian hardly ever supplies an adequate approximation to van der Waals or to Casimir forces even when all bodies are at the same temperature.

The very severe limitations of LTE for the classical problem have been documented elsewhere [3]; so has the necessity for any such discussions to spell out, at least in principle, the operation of the thermostats that constrain different parts of the system to remain at different temperatures. Here we extend the theory to identical Ohmically damped quantum oscillators at arbitrary $T_{1}$ and $T_{2}$, i.e. below the classical high-temperature limit. Either oscillator when isolated has undamped natural frequency $\omega_{0}$ and damping constant (inverse mean life) $\eta$. The exercise is nontrivial because it must adopt, and explicate, a model of continuously extended systems that double as the heat baths responsible for the damping, and as the thermostats enforcing the nominally prescribed temperatures $T_{1}$ and $T_{2}$. Though we find integrals expressing $\mathcal{P}$ for any combinations of temperatures and for any $g \equiv \eta / \omega_{0}$, we aim to generate insight rather than to facilitate numerics; and to this end concentrate chiefly on the common case of weak (far-subcritical) damping $g \ll 1$, and on relatively transparent approximations appropriate when $k_{B} T_{1,2}$ are either well below or well above $\hbar \omega_{0}$.

\subsection{The Model}

Each oscillator has its own heat bath, acting also as its thermostat. Quā heat-bath its spectrum is determined by our requirement that the damping it produces be Ohmic, and the simplest way to ensure this is to follow Unruh and Zurek [25] by modelling it as a string stretched from $x=-\infty$ to $x=0$, attached to the oscillator at $x=0$. We write the string mass per unit length as $\sigma$, the wave speed along the string as $c$, and choose natural units such that $c=1$. (Since we focus on the differences between quantum and classical behaviour, we emphatically do not set $\hbar=1$.) The velocity potential for points on the string is $\tilde{\psi}(x, t)$, and the force $\sigma \partial \tilde{\psi} / \partial t$ : see e.g. Sect. 10.1 in [2]. The oscillator displacement is $\tilde{q}$, so that $-\partial \tilde{\psi}(x, t) /\left.\partial x\right|_{x=0}=d \tilde{q} / d t$. The primary dynamical variables $\tilde{q}$ and $\tilde{\psi}$ carry tildes in order to free $q \equiv \sqrt{m} \tilde{q}$ and $\psi \equiv \sqrt{\sigma} \tilde{\psi}$ for more conveniently normed versions to be motivated presently in Sect. 2. Integrals $\int d x \ldots$ are short for $\int_{-\infty}^{0+} d x \ldots$, with $\delta(x)$ inside the integration range.

In order to make the bath double as a thermostat, constraining the oscillator as closely as possible to a preassigned temperature $T$, we picture it as attached at $x=-\infty$ to an external device which forces the string to execute temperature- $T$ wave motion identical to what it would execute if the end at $x=0$ were fixed, in addition to whatever motions are generated by the oscillator. What the oscillator then does must be found by solving the equations of motion. One could of course try to pursue the further question, already raised in [3], how such a device, call it the first thermostat, might be kept functioning as envisaged; to explore this would then entail hypothesising a second thermostatting device controlling the first, and calculating the behaviour of the first instead of making assumptions about it a priori; and so on, potentially ad infinitum. In the end, one must necessarily settle for control at some level through thermostats that are external to the system in the sense that they impose temperatures by fiat, their own dynamics not entering the calculation and not needing to be spelled out. 
The point is that our assumptions must not be selfcontradictory at the level where they are to operate: that is the trap we avoid by assigning definite temperatures to the thermostats, rather than to each oscillator in all respects and in its entirety.

By contrast to such nonequilibrium steady states, given overall thermal equilibrium there is no need to solve equations of motion in order to find, say, the mean-square displacements of any number of oscillators coupled to possibly different heat baths, as well as to each other. Then, given a sensible Hamiltonian for the oscillators and the baths, including all their interactions, one proceeds from the canonical distribution for the entire system, as is done for a single oscillator in the classic papers [6,7]. Section 5.2.1 will confirm that the results are the same either way.

\subsection{Preview and Summary}

Part I (Sects. 2-5) uses our model to determine the mean-square displacement $\left\langle q^{2}\right\rangle \equiv$ $\lim _{t \rightarrow \infty}\left\langle q^{2}(t)\right\rangle$ of a single thermostatted oscillator in its ultimate steady state, and verifies that, whatever the initial conditions, it is the same as it would be in overall thermal equilibrium of string plus oscillator, understood conventionally, with no thermostatting mechanism needing to be prescribed. The thermal-equilibrium results for Ohmic damping are common knowledge (much of it from the splendid analysis by Grabert et al. [15]); but we must look at them in some detail, because part II uses them to study two mutually coupled oscillators maintained at different temperatures by attaching each to its own string serving as a thermostat. In particular we determine the heat-flow under these conditions, governed by the quantum-mechanical generalization of the purely classical model used in [3].

In Part I, Sects. 2 and 3 derive the canonical equations of motion of coupled string and oscillator. (For convenience, Appendix 2 adapts the standard expansion of the free quantized 1D scalar field to our half-line.) Section 4.1 takes the crucial step of splitting solutions for the string into a particular integral embodying its response to the oscillator, plus a complementary function empowering it as a thermostat. Section 4.2 identifies the quantum Langevin force $f(t)$ experienced by the oscillator; verifies that the damping is indeed Ohmic; and writes down its susceptibility $\chi(\omega)$, Eq. (4.10), whose denominator $D^{2}\left(u \equiv \omega / \omega_{0}, g\right)$, with its remarkable integrals (Appendix 1), features prominently throughout the paper. Section 4.3 then finds the correlation function $F\left(t-t^{\prime}\right)$ between $f(t)$ and $f\left(t^{\prime}\right)$, Eq. (4.19), and determines its Fourier transform $\tilde{F}(\omega)$, Eq. (4.22), which is central to most of what follows. (For completeness, the properties of $F\left(t-t^{\prime}\right)$ itself are summarized in Appendix 3.)

Section 5.1 studies the evolution of the oscillator coordinates $q(t)$ and $\dot{q}(t)$. Section 5.2.1 calculates the steady-state limit $\left\langle q^{2}\right\rangle$; Sect. 5.2.2 approximates it in the high-temperature (near-classical) regime $k_{B} T \gg h \omega_{0}$, Eq. (5.12); finally Sect. 5.2.3 derives approximations in the essentially quantal low-temperature regime $k_{B} T \ll h \omega_{0} .{ }^{1}$ The awkwardness here stems from the fact that the classic zero-temperature integral $\digamma(g)$, Eq. (5.13), conceals an arctangent which must be pursued over three of its branches.

In Part II, Sect. 6.1 introduces, for two oscillators 1 and 2 having internal coordinates $q_{1,2}$ and dimensionless mutual coupling constant $\lambda$, the normal-mode coordinates $X=\left(q_{1}+q_{2}\right) / 2$ and $\xi=\left(q_{1}-q_{2}\right)$; their natural frequencies $\omega_{p, n}=\omega_{0} \sqrt{1 \pm \lambda}$; the associated Langevin forces when the oscillators are thermostatted to $T_{1}, T_{2}$ respectively; and it shows that the heat flow from 1 to 2 is $\mathcal{P}=\lambda \omega_{0}^{2}\langle X \dot{\xi}\rangle$. To gain some preliminary orientation,

1 Section 8.1.2 will explain that if $\omega_{0}$ is in the visible, appropriately to electronic excitations, then practicable scenarios realize the low-temperature regime, because, unless $k_{B} T / h \omega_{0} \ll 1$, atoms are mostly ionized, and solids close to melting. But there is no such general rule if $\omega_{0}$ is in the infrared, appropriately to molecular or to solid-state excitations. 
Sect. 6.2 then quotes from [3] the classical steady-state expectation values $\left\langle X^{2}, \xi^{2}, X \xi, X \dot{\xi}\right\rangle_{c l}$, Eqs. $(6.16,6.17)$; comments briefly on the problems attending LTE; and makes what comparisons are possible with other theoretical work, and with measurements on coupled electric circuits.

Section 7.1 determines the crucial correlator $\langle X \dot{\xi}\rangle$ exactly, Eqs. (7.10-7.12), and finds relatively transparent approximations at high temperatures, Eq. (7.14), and at low temperatures, Eqs. (7.15-7.17) plus (7.20). In particular, Sect. 8.3 will use (7.14) to confirm the high-temperature limit from [3], namely $\mathcal{P}_{c l}=\left[g \lambda^{2} / 2\left(\lambda^{2}+g^{2}\right)\right] \omega_{0} k_{B}\left(T_{1}-T_{2}\right)$; and will use (7.20) to show that low $T_{1}$ and $T_{2}$ make $\mathcal{P}$ proportional to $\left[\lambda^{2} g^{2} /\left(1-\lambda^{2}\right)^{2}\right]\left[\left(k_{B} T_{1}\right)^{4}-\right.$ $\left.\left(k_{B} T_{2}\right)^{4}\right] /\left(\hbar \omega_{0}\right)^{4}$. Section 7.2 ties LTE to perturbation theory to leading order in $\lambda$, serving also as a partial check on the laborious and thereby accident-prone derivations of the exact results.

Section 8.1 proposes the estimate $|\lambda| \sim 1 / r^{3}$ as roughly appropriate to electric-dipole coupling between neutral atoms or molecules separated by a distance $r$ in atomic units; and, for the dimensionless dissipation parameter $g$, the orders of magnitude $g \sim \mathcal{O}\left(10^{-7}\right)$ if the damping is radiative (and hence, strictly speaking, beyond the remit of our model), or $g \sim 10^{-1}$ to $10^{-3} \ll 1$, if it is to be comparable with Ohmic damping in common macroscopic materials. On this basis, Sect. 8.2 considers the two leading terms of the lowtemperature approximation to $\mathcal{P}$; Sect. 8.3 constructs plausible estimates of its dependence on $T_{1}$ and $T_{2}$, Eq. (8.12), as already anticipated above; and Sect. 8.4 estimates its dependence on distance, namely $\mathcal{P} \propto g^{2} / r^{6}$, Eq. (8.16).

Finally, and just as an illustration, Appendix 4 considers two identical Drude-modelled half-spaces of insulators a distance $Z$ apart, each with plasma frequency $\omega_{p}$, damping constant $\Gamma$, and undamped surface-plasmon frequency $\omega_{S}=\sqrt{\omega_{0}^{2}+\omega_{p}^{2} / 2}$ independent of the surface wave-vector $\mathbf{k}$. Generalizing the classical calculation in [3], we find that the heat flux $\mathcal{R}$ between them is given by the integral (12.3) of $\mathcal{P}$ over $\mathbf{k}$, with $\omega_{0} \rightarrow \omega_{S}$ and $\lambda \rightarrow \beta_{S}^{2} \exp (-k Z)$, where $\beta_{S}^{2}=\omega_{p}^{2} / 2 \omega_{S}^{2}$, Eq. (12.2). At low temperatures Eq. (12.15) makes $\mathcal{R}$ proportional to $-\log \left(1-\beta_{S}^{4}\right)\left(\Gamma^{2} / Z^{2}\right)\left[\left(k_{B} T_{1}\right)^{4}-\left(k_{B} T_{2}\right)^{4}\right] /\left(\hbar \omega_{S}\right)^{4}$. Unfortunately, at low temperatures this approach is unsuited to conductors, which have $\omega_{0}=0$ and $\beta_{S}^{2}=1$.

\section{Part I: Single Oscillator}

Section 1.2 has explained that the nonequilibrium steady-state properties of our model must be found by solving its equations of motion; and why, as a preliminary, Part I does this in order to find the mean-square displacement of a single oscillator (even though it is already well-known from the true equilibrium state). We use the Euler-Lagrange equations derived from the Lagrangean $\mathcal{L}$ constructed in Sect. 2, featuring the dynamical variables and the parameters already defined in Sects. 1.1 and 1.2.

\section{Canonicals}

We work in what QED would call the $\mathbf{E} \cdot \mathbf{r}$ gauge. Then the Lagrangean reads

$$
\begin{aligned}
\mathcal{L} & =m \tilde{q} / 2-m \omega_{0}^{2} \tilde{q}^{2} / 2+\int d x\left\{\sigma \tilde{\psi} / 2-\sigma(\partial \tilde{\psi} / \partial x)^{2} / 2+\delta(x) \tilde{q} \tilde{\psi}(x)\right\} \\
& =m \dot{\tilde{q}}^{2} / 2-m \omega_{0}^{2} \tilde{q}^{2} / 2+\int d x\left\{\sigma \tilde{\psi} / 2-\sigma(\partial \tilde{\psi} / \partial x)^{2} / 2\right\}+\left.\tilde{q} \tilde{\psi}\right|_{x=0}
\end{aligned}
$$


The first term on the right is the kinetic and the second the potential energy of the oscillator. Under the integral, the first and the second terms are the kinetic and the potential energies per unit length of the string. The final term on the right of either line is the interaction energy between string and oscillator.

However, it proves convenient to change to scaled variables (without tildes), namely to

$$
q \equiv \sqrt{m} \tilde{q}, \quad \psi \equiv \sqrt{\sigma} \tilde{\psi}, \quad \mu \equiv 1 / \sqrt{m \sigma},
$$

so that

$$
\begin{aligned}
\mathcal{L} & =\dot{q}^{2} / 2-\omega_{0}^{2} q^{2} / 2+\int d x\left\{\dot{\psi}^{2} / 2-(\partial \psi / \partial x)^{2} / 2+\delta(x) \mu q \dot{\psi}(x)\right\} \\
& =\dot{q}^{2} / 2-\omega_{0}^{2} q^{2} / 2+\int d x\left\{\dot{\psi}^{2} / 2-(\partial \psi / \partial x)^{2} / 2\right\}+\left.\mu q \dot{\psi}\right|_{x=0} .
\end{aligned}
$$

This is the form of the Lagrangean that we shall use. The canonical momenta are

$$
p=\partial \mathcal{L} / \partial \dot{q}=\dot{q}, \quad \Pi(x)=\delta \mathcal{L} / \delta \dot{\psi}=\dot{\psi}=+\mu q \delta(x) .
$$

We indicate physical dimensions by square brackets [...], with [length, time, mass] $=[\mathrm{L}$, $\mathrm{T}, \mathrm{M}]$; use (unrationalized) Gaussian units, so that $\left[\right.$ charge $\left.{ }^{2}\right]=\left[\mathrm{ML}^{3} \mathrm{~T}^{-2}\right]$; and note that

$$
[q]=M^{1 / 2} T, \quad[p]=M^{1 / 2}, \quad[\psi]=M^{1 / 2} T^{1 / 2}, \quad[\Pi]=M^{1 / 2} T^{-1 / 2}, \quad[\mu]=T^{-1 / 2} .
$$

In natural units, with $c=1$, one checks dimensions as if $[L]=[T]$.

Corresponding to $\mathcal{L}$ one has the Hamiltonian

$$
\begin{aligned}
\mathcal{H}= & p \dot{q}+\int d x \Pi \dot{\psi}-\mathcal{L}=p^{2} / 2+\omega_{0}^{2} q^{2} / 2+\int d x\left\{\Pi^{2} / 2+(\partial \psi / \partial x)^{2} / 2\right\}-\mu q \Pi(t, 0) \\
& +\mu^{2} q^{2} \delta(0) / 2 .
\end{aligned}
$$

Expressed noncanonically,

$$
\mathcal{H}=\dot{q}^{2} / 2+\omega_{0}^{2} q^{2} / 2+\int d x\left\{\dot{\psi}^{2} / 2+(\partial \psi / \partial x)^{2} / 2\right\},
$$

showing that $\mathcal{H}$ is nonnegative, whence the system is stable.

The divergent last term of (2.6) indicates that our model is unphysical in that it requires an infinite amount of energy to connect the oscillator to the string. Often (if implicitly) the divergence is cured by imposing a frequency cutoff on the normal modes from Sect. 3 below, the same as the cutoff required at the end of Sect. 5 in order to yield finite expectation values of $\dot{q}^{2}$. However, even though the divergent term in (2.6) looks like a squared-frequency shift $\omega_{0}^{2} \rightarrow\left[\omega_{0}^{2}+\mu^{2} \delta(0)\right]$, we shall see presently that all pertinent Green's functions and other expressions feature $\omega_{0}^{2}$ unshifted. This tallies with Eq. (4.9) of Caldeira and Leggett [6], which in common parlance says that our model does not renormalize the frequency, ${ }^{2}$ i.e. that it has $\omega_{R}=\omega_{0}$ : see for instance the phase shift $\vartheta$ in (3.6), the denominator $D^{2}$ in (3.7), and thereby the expectation value of $q^{2}$ in (5.10). However, one must not read too much into this persistence of $\omega_{0}$ in our equations: for instance, it has no direct bearing on the spectral

2 In fact, the present writer thinks that to the kind of problem we are considering the concept of "renormalization" is inappropriate, because the input parameter $\omega_{0}$ is measurable by decoupling the oscillator from the thermostat. The only exception to this rule would seem to be QED, where the thermostat is the quantized Maxwell field, from which nothing can decouple even at absolute zero. 
weights of the exact normal modes in thermal equilibrium, say as studied by Barnett et al. [1] and by Philbin and Anders [24].

The equal-time commutators are

$$
[q(t), p(t)]=i \hbar, \quad[\psi(t, x), \Pi(t, y)]=i \hbar \delta(x-y) .
$$

For completeness, we note that our $\mathbf{E} \cdot \mathbf{r}$ gauge is linked to the analogue of the $\mathbf{A} \cdot \mathbf{p}$ gauge by a unitary transformation $U$ :

$U=\exp \left(i \mu \psi_{0} q / \hbar\right): \quad U\{q, p, \psi, \Pi\} U^{-1}=\left\{q^{\prime}, p^{\prime}, \psi^{\prime}, \Pi^{\prime}\right\}=\left\{q, p-\mu \psi_{0}, \Pi-\delta(x) \mu q\right\}$,

$$
U \mathcal{H} U^{-1}=\mathcal{H}^{\prime}=\left(p^{\prime}+\mu \psi_{0}\right)^{2} / 2+\omega_{0}^{2} q^{2} / 2+\int d x\left\{\Pi^{\prime 2} / 2+(\partial \psi / \partial x)^{2} / 2\right\} .
$$

\section{The Equations of Motion}

The Euler-Lagrange equations read

$$
\begin{aligned}
& 0=\frac{d}{d t}\left(\frac{\partial \mathcal{L}}{\partial \dot{q}}\right)-\frac{\partial \mathcal{L}}{\partial q} \Rightarrow \ddot{q}+\omega_{0}^{2} q=\mu \dot{\psi}(t, 0), \\
& 0=\frac{d}{d t}\left(\frac{\delta \mathcal{L}}{\delta \dot{\psi}}\right)+\frac{\partial}{\partial x}\left(\frac{\delta \mathcal{L}}{\delta(\partial \psi / \delta x)}\right) \Rightarrow \ddot{\psi}-\frac{\partial^{2} \psi}{\partial x^{2}}=-\mu \dot{q} \delta(x) .
\end{aligned}
$$

To ease cross-referencing to displayed equations embracing more than one equality we shall indicate the first such equality with a label a appended to the equation number, the second with a label b, and so on. For instance, the rightmost equality in (3.2) can be cited as (3.2)b.

Using this notation, we note that the awkward right-hand side of (3.2)b can be eliminated in favour of a boundary condition. For brevity, define

$$
\psi_{0}(t) \equiv \psi(x=0-, t), \quad \frac{\partial \psi_{0}(t)}{\partial x} \equiv \frac{\partial \psi}{\partial x}(x=0-, t) ;
$$

then act on (3.2)b with $\int_{0-}^{0+} d x \ldots$, use $[\psi(x>0)=0] \Rightarrow\left[\partial \psi /\left.\partial x\right|_{x=0-}=0\right]$, and find

$$
x<0: \quad \ddot{\psi}-\partial^{2} \psi / \partial x^{2}=0, \quad \partial \psi_{0} / \partial x=-\mu \dot{q} .
$$

Without the oscillator, i.e. if $\mu=0$, this implies a free field under a Neumann boundary condition at the origin, as outlined in Appendix 2.

The present paper focusses on the oscillator, whose behaviour, however, may be easier to appreciate if, as a preliminary exercise, one applies the equations of motion to determine to normal modes of the string regarded as an object of interest in its own right, with the oscillator merely responsible for the boundary condition (3.4)b. All one needs are the coupled solutions of (3.1)b and (3.2)b proportional to exp $(-i \omega t)$. Dropping this common factor, admitting an overall norming constant $A$, and defining

$$
\eta \equiv \mu^{2}, \quad[\eta]=T^{-1} .
$$

it easily verified that

$$
\psi(x<0)=A \cos [\omega x+\vartheta(\omega)], \quad q=A \exp (i \pi / 2) \sin (\vartheta) / \mu, \quad \tan (\vartheta)=\frac{\eta \omega}{\left(\omega_{0}^{2}-\omega^{2}\right)} .
$$


The phase shift $\vartheta(\omega)$ vanishes at $\omega=0$, rises monotonically through a resonance at the natural frequency of the oscillator, i.e. through $\vartheta\left(\omega_{0}\right)=\pi / 2$, and then rises to $\pi$ as $\omega \rightarrow \infty$.

With an eye on $\sin ^{2}(\vartheta)=\tan ^{2}(\vartheta) /\left[1+\tan ^{2}(\vartheta)\right]$, we define dimensionless

$$
u \equiv \omega / \omega_{0}, \quad g \equiv \eta / \omega_{0}, \quad D^{2}(u, g) \equiv u^{4}-\left(2-g^{2}\right) u^{2}+1=\left(1-u^{2}\right)^{2}+g^{2} u^{2}
$$

then $\sin ^{2}(\vartheta)=\left(u^{2} g^{2}\right) / D^{2}$, and $q=A \exp (i \pi / 2)\left(\mu \omega_{0}\right) u / D$. Important properties of the function $1 / D^{2}$ are collected in Appendix 1. The reflection coefficient $R$ is

$$
R=\exp (-2 i \vartheta)=\frac{1-i \tan (\vartheta)}{1+i \tan (\vartheta)}=\frac{\omega_{0}^{2}-\omega^{2}-i \eta \omega}{\omega_{0}^{2}-\omega^{2}+i \eta \omega}=\frac{1-u^{2}-i g u}{1-u^{2}+i g u}
$$

\section{Solutions}

\subsection{Strategy}

Rearranged for convenience, the equations of motion (3.1) and (3.2) read

$$
x<0: \quad \ddot{\psi}-\frac{\partial^{2} \psi}{\partial x^{2}}=0, \quad \frac{\partial \psi_{0}}{\partial x}=-\mu \dot{q} ; \quad \ddot{q}+\omega_{0}^{2} q=\mu \dot{\psi}_{0} .
$$

Both are inhomogeneous, the first on account of the boundary condition (4.1)b, and the second on account of $\mu \dot{\psi}_{0}$ on its right. We shall deal with each inhomogeneity by the standard trick of pretending that it is known in advance.

Starting with the field, we split

$$
\psi=\Phi+\phi
$$

where $\Phi$ is a particular integral and $\phi$ a complementary function. They are chosen so that

$$
\begin{aligned}
& \ddot{\Phi}-\frac{\partial^{2} \Phi}{\partial x^{2}}=0, \quad \frac{\partial \Phi_{0}}{\partial x}=-\mu \dot{q}, \quad \Phi(t=0, x)=0, \quad \dot{\Phi}(t=0, x)=0, \\
& \ddot{\phi}-\frac{\partial^{2} \phi}{\partial x^{2}}=0, \quad \frac{\partial \phi_{0}}{\partial x}=0, \quad \phi(0, x)=\psi(0, x), \quad \dot{\phi}(0, x)=\dot{\psi}(0, x) .
\end{aligned}
$$

Following (3.3) we have written $\phi_{0} \equiv \phi(x=0), \partial \phi_{0} / \partial x \equiv \partial \phi_{0} /\left.\partial x\right|_{x=0}$, etc.

The particular integral $\Phi$ is to be generated causally by the oscillator, via (4.1)b, so that

$$
\mu \frac{\partial \Phi_{0}}{\partial t}=-\mu^{2} \dot{q}=-\eta \dot{q} .
$$

By contrast, $\phi(t, x)$ knows nothing about the oscillator: it is simply a free field subject to a Neumann boundary condition, but features jointly with $\Phi$ in the equation of motion (4.1)c for $q$. As explained in Sect. 1, we model our thermostat by identifying $\phi$ with the free quantized field appropriate to the temperature $T$ we wish to prescribe for the oscillator. The basics of the free field on a half-line are spelled out in Appendix 2.

To summarize, roughly speaking $\phi$ drives while $\Phi$ damps. 


\subsection{Ohmic Damping}

To solve (4.3) one uses the causal Neumann Green's function $G_{N}$ and the standard rule applicable to a prescribed derivative on the boundary ([2, Sects. 10.4 and 12.8]):

$$
\begin{aligned}
G_{N}\left(x, t \mid x^{\prime}, t^{\prime}\right) & =\theta\left(t-t^{\prime}\right) \frac{1}{2}\left\{\theta\left(t-t^{\prime}-\left|x-x^{\prime}\right|\right)+\theta\left(t-t^{\prime}+\left|x-x^{\prime}\right|\right)\right\}, \\
\Phi(t, x) & =\int_{0}^{t} d t^{\prime} G_{N}\left(x, t \mid 0, t^{\prime}\right)\left[-\mu \dot{q}\left(t^{\prime}\right)\right]=-\mu \int_{0}^{t-|x|} d t^{\prime} \dot{q}\left(t^{\prime}\right) \\
& =-\mu[q(t-|x|)]-q(0),
\end{aligned}
$$

where $\theta$ is the Heaviside step function. Hence

$$
\dot{\Phi}(t, 0)=-\mu \dot{q}(t), \quad \dot{\psi}(t, 0)=-\mu \dot{q}(t)+\mu \dot{\phi}(t, 0),
$$

and substitution into (3.1)b yields

$$
\ddot{q}+\eta \dot{q}(t)+\omega_{0}^{2} q=f(t), \quad \eta=\mu^{2}, \quad f(t) \equiv \mu \dot{\phi}(t, 0), \quad[f]=M^{1 / 2} T^{-1} .
$$

This identifies $\eta$ as the Ohmic (Markovian) damping constant, and $f(t)$, to be discussed in the next section, as the randomly fluctuating Langevin force familiar from Brownian motion. We recall the dynamic polarizability $\chi$ of the Ohmically damped simple-harmonic oscillator,

$$
\begin{aligned}
& \chi(\omega)=\frac{\chi_{0} \omega_{0}^{2}}{\omega_{0}^{2}-\omega^{2}-i \eta \omega}=\frac{\chi_{0}}{\left[1-u^{2}-i g u\right]}=\frac{\chi_{0}\left[1-u^{2}+i u g\right]}{D^{2}(u, g)}, \\
& \operatorname{Im}\left[\frac{\chi}{\chi_{0}}\right]=\frac{u g}{D^{2}(u, g)}, \quad \chi_{0}=\frac{e^{2}}{m \omega_{0}^{2}},
\end{aligned}
$$

linked to the reflection amplitude (3.8) by $R=\chi(\omega) / \chi^{*}(\omega)=\chi(\omega) / \chi(-\omega)$.

The normal modes and the eigenfrequencies of the damped but unforced oscillator, i.e. of (4.9)a in absence of $f(t)$, read

$$
\begin{gathered}
q(t)=q(0) \exp (-i \Omega t), \quad \Omega^{2}+i \eta \Omega-\omega_{0}^{2}=0 \\
\Omega=-i \eta / 2 \pm \sqrt{\omega_{0}^{2}-\eta^{2} / 4} \equiv \Omega_{ \pm}, \quad \Omega_{ \pm}^{2}=\omega_{0}^{2}-\eta^{2} / 2 \mp i \eta \sqrt{\omega_{0}^{2}-\eta^{2} / 4}
\end{gathered}
$$

Appendix 1 shows that $\Omega_{ \pm}=\omega_{0} u_{ \pm}$, where $\pm u_{+}$and $\pm u_{-}$are the four roots of $D^{2}(u, g)$ from (3.7). In view of (4.13) we define

$$
\Omega_{0} \equiv \sqrt{\omega_{0}^{2}-\eta^{2} / 4}, \quad \omega_{0}^{2}=\Omega_{0}^{2}+\eta^{2} / 4 .
$$

For simplicity we shall, generally, display our results in the underdamped scenario $\eta^{2}<4 \omega_{0}^{2}$, where $\Omega_{0}$ is real. Overdamping can be dealt with more or less straightforwardly by analytic continuation.

To deal with (4.9)a, Sect. 5 will need its causal Green's function, defined and given by

$$
\begin{aligned}
& {\left[\partial^{2} / \partial t^{2}+\eta \partial / \partial t+\omega_{0}^{2}\right] \mathcal{G}\left(t, t^{\prime}\right)=\delta\left(t-t^{\prime}\right), \quad \mathcal{G}\left(t<t^{\prime}\right)=0,} \\
& \mathcal{G}\left(t, t^{\prime}\right)=\theta\left(t-t^{\prime}\right) \frac{1}{\Omega_{0}} \exp \left[-\eta\left(t-t^{\prime}\right) / 2\right] \sin \left[\Omega_{0}\left(t-t^{\prime}\right)\right] .
\end{aligned}
$$




\subsection{The Langevin Force}

From the definition (4.9)c of $f$ and from Appendix 2 for $\phi$ we derive the commutators

$$
\begin{aligned}
& f\left(t^{\prime}\right) f\left(t^{\prime \prime}\right)-f\left(t^{\prime \prime}\right) f\left(t^{\prime}\right)=-\frac{2 i \eta}{\pi} \int_{0}^{\infty} d \omega \hbar \omega \sin (\omega \tau)=2 i \eta \hbar \frac{\partial}{\partial \tau} \delta(\tau), \quad \tau=t^{\prime}-t^{\prime \prime}, \\
& f\left(t^{\prime}\right) \dot{f}\left(t^{\prime \prime}\right)-\dot{f}\left(t^{\prime \prime}\right) f\left(t^{\prime}\right)=\frac{2 i \eta}{\pi} \int_{0}^{\infty} d \omega \hbar \omega^{2} \cos (\omega \tau)=-2 i \eta \hbar \frac{\partial^{2}}{\partial \tau^{2}} \delta(\tau),
\end{aligned}
$$

and shall need also the correlator

$$
F(\tau) \equiv \frac{1}{2}\left\langle f\left(t^{\prime}\right) f\left(t^{\prime \prime}\right)+f\left(t^{\prime \prime}\right) f\left(t^{\prime}\right)\right\rangle, \quad[F]=M T^{-2},
$$

where $\langle\ldots\rangle$ stands for the expectation value in thermal equilibrium. By (4.9)c, (10.3), and

$$
n(\omega) \equiv 1 /[\exp (\beta \hbar \omega)-1], \quad \beta \equiv 1 / k_{B} T,
$$

one has

$$
\begin{aligned}
& F(\tau)=\int_{-\infty}^{\infty} d \omega \tilde{F}(\omega) \cos (\omega \tau)=\int_{-\infty}^{\infty} d \omega \tilde{F}(\omega) \exp (i \omega \tau) \\
& \tilde{F}(\omega)=\frac{\eta \hbar \omega}{2 \pi}[2 n(\omega)+1]=\frac{\eta \hbar \omega}{2 \pi} \operatorname{coth}\left(\frac{\beta \hbar \omega}{2}\right), \quad[\tilde{F}]=M T^{-1}
\end{aligned}
$$

We recall the expansions

$$
\operatorname{coth}(x)=\operatorname{sign}(x)\left[\frac{1}{|x|}+\frac{|x|}{3}-\frac{|x|^{3}}{45}+\cdots\right]=\operatorname{sign}(x)\left[1+2 \sum_{n=1}^{\infty} \exp (-2 n|x|)\right],
$$

holding, respectively, for $|x|<\pi$ and for arbitrary $x$.

Appendix 3 explores $F(\tau)$, mainly from curiosity; but the bulk of our calculations use the Fourier transform $\tilde{F}(\omega)$, and we note at once its classical/high-temperature and its zerotemperature limits:

$$
\begin{gathered}
\tilde{F}_{c l}(\omega) \equiv \tilde{F}(\beta \hbar \omega \rightarrow 0)=\frac{\eta}{\pi \beta} \Rightarrow F_{c l}(\tau)=\frac{2 \eta}{\beta} \delta(\tau), \\
\tilde{F}_{0}(\omega) \equiv \tilde{F}(\beta \hbar|\omega| \rightarrow \infty)=\frac{\eta|\omega|}{2 \pi} \Rightarrow F_{0}(\tau)=-\frac{\eta \hbar}{\pi \tau^{2}} .
\end{gathered}
$$

\section{$5 q$ and $\dot{q}$ in the Steady State}

The chief object of this section is to derive $(5.10,5.11)$ for the exact steady-state meansquare displacement; its high- $T$ asymptotics (5.12); and its low- $T$ asymptotics (5.13-5.16) plus (5.22).

\section{$5.1 q(t)$ and $\dot{q}(t)$}

The complete solution of (4.9)a for $q$, like that of (4.1)a,b for $\psi$, splits into a particular integral, plus a complementary function which accommodates the commutation rules and 
the initial conditions on $q$ and $\dot{q}$. We drop the complementary function because it vanishes as $t \rightarrow \infty$, i.e. in the final steady state; in other words, ultimately the initial conditions prove irrelevant. Meanwhile, for finite $t$ our expressions apply subject to $q(0)=0=\dot{q}(0)$. Then

$$
q(t)=\int_{0}^{t} d t^{\prime} \mathcal{G}\left(t, t^{\prime}\right) f\left(t^{\prime}\right)=\int_{0}^{t} d \tau \mathcal{G}(\tau) f(t-\tau), \quad \tau \equiv t-t^{\prime},
$$

where, by (4.16),

$$
\begin{aligned}
& \mathcal{G}(\tau)=\frac{1}{\Omega_{0}} \exp (-\eta \tau / 2) \sin \left(\Omega_{0} \tau\right), \\
& \mathcal{G}(0)=\mathcal{G}(\infty)=0, \quad \partial \mathcal{G} /\left.\partial \tau\right|_{\tau=0}=1 .
\end{aligned}
$$

Finally, by (5.1)a and (5.2)b

$$
\dot{q}(t)=\int_{0}^{t} d t^{\prime} \frac{\partial \mathcal{G}\left(t, t^{\prime}\right)}{\partial t} f\left(t^{\prime}\right)=\int_{0}^{t} d \tau \frac{\partial G(\tau)}{\partial \tau} f(t-\tau) .
$$

\subsection{The Steady-State Mean-Square Displacement $\left\langle q^{2}\right\rangle$}

We abbreviate the steady-state limit as

$$
\left\langle q^{2}\right\rangle \equiv \lim _{t \rightarrow \infty}\left\langle q^{2}(t)\right\rangle
$$

In the present case of a single oscillator $\left\langle q^{2}\right\rangle$ is expected and will turn out to be the muchdiscussed thermal-equilibrium value. To determine it according to our model we generalize the classical calculation in Sect. 3 of [3].

\subsection{1 $\left\langle q^{2}\right\rangle$ Exactly}

In virtue of (5.1)

$$
\left\langle q^{2}\right\rangle \equiv \lim _{t \rightarrow \infty}\left\langle q^{2}(t)\right\rangle=\int_{0}^{\infty} \int_{0}^{\infty} d \tau^{\prime} d \tau^{\prime \prime} \mathcal{G}\left(\tau^{\prime}\right) \mathcal{G}\left(\tau^{\prime \prime}\right)\left\langle F\left(\tau^{\prime}-\tau^{\prime \prime}\right)\right\rangle
$$

hence, by (5.2)

$$
\begin{aligned}
\left\langle q^{2}\right\rangle & =\frac{1}{\Omega_{0}^{2}} \int_{-\infty}^{\infty} d \omega \tilde{F}(\omega)\left|\int_{0}^{\infty} d \tau \sin \left(\Omega_{0} \tau\right) \exp [-\tau(\eta / 2+i \omega)]\right|^{2} \\
& =\frac{1}{\Omega_{0}^{2}} \int_{-\infty}^{\infty} d \omega \tilde{F}(\omega)\left|\frac{\Omega_{0}}{\Omega_{0}^{2}+(\eta / 2+i \omega)^{2}}\right|^{2}=\int_{-\infty}^{\infty} d \omega \tilde{F}(\omega) \frac{1}{\left[\left(\omega_{0}^{2}-\omega^{2}\right)^{2}+\eta^{2} \omega^{2}\right]} .
\end{aligned}
$$

One takes $\tilde{F}(\omega)$ from (4.22), scales to dimensionless $u \equiv \omega / \omega_{0}$ and $g \equiv \eta / \omega_{0}$ from (3.7), defines likewise dimensionless

$$
\alpha \equiv \hbar \omega_{0} \beta / 2=\hbar \omega_{0} / 2 k_{B} T
$$

and obtains

$$
\begin{aligned}
\left\langle q^{2}\right\rangle & =\frac{\hbar}{2 \pi \omega_{0}} \int_{-\infty}^{\infty} d u \frac{g u \operatorname{coth}(\alpha u)}{D^{2}(u, g)}=\frac{\hbar}{\omega_{0}} \mathcal{K}(\alpha, g), \\
\mathcal{K} & \equiv \frac{1}{2 \pi} \int_{-\infty}^{\infty} d u \frac{g u \operatorname{coth}(\alpha u)}{D^{2}(u, g)}=\frac{1}{2 \pi} \int_{-\infty}^{\infty} d u \operatorname{Im}\left[\frac{\chi(\omega)}{\chi_{0}}\right] \operatorname{coth}(\alpha u),
\end{aligned}
$$


featuring the oscillator polarizability $\chi$ from $(4.10,4.11)$. It is straightforward to check that (5.8) and (5.11) tally with, say, the classic results (6.35) in Caldeira and Leggett [6] and (B.9) in Caldeira and Leggett [7], and with (2.17) in Grabert et al. [15] (where $t \rightarrow 0$ and $\gamma \rightarrow \eta$ ).

We proceed to look for transparent approximations first in the classical regime appropriate, formally, to $\hbar \rightarrow 0$, or more realistically to high enough temperatures; and then in the quantum regime appropriate, formally, to $\hbar \rightarrow \infty$, or more realistically to low enough temperatures. To improve on such asymptotics one would have to evaluate $\mathcal{K}$ exactly, closing the integration contour at infinity say in the upper-half complex $u$ plane, and picking up the residues from the Matsubara poles of coth $(\alpha u)$ as well as those from the poles of $1 / D^{2}(u, g)$. Equation (4.4) of [15] spells out the exact result as a closed-form though somewhat convoluted expression in terms of digamma functions.

\subsubsection{The Near-Classical Regime}

The classical limit $\alpha \rightarrow 0$ approached under the integral in (5.11) invites one to use as much of (4.23)a as convergence at infinity permits, namely the first two terms. Then, by (9.7),

$$
\begin{aligned}
\alpha \rightarrow 0: \quad \mathcal{K} & \simeq \frac{g}{2 \pi} \int_{-\infty}^{\infty} d u \frac{u}{D^{2}(u, g)}\left\{\frac{1}{\alpha u}+\frac{\alpha u}{3}\right\}=\frac{1}{2}\left\{\frac{1}{\alpha}+\frac{\alpha}{3}\right\} \\
& \Rightarrow\left\langle q^{2}\right\rangle_{c l} \simeq \frac{k_{B} T}{\omega_{0}^{2}}+\frac{\hbar^{2}}{12 k_{B} T} .
\end{aligned}
$$

Neither term features $g$ : to this order one gets the same as for an undamped oscillator. A better approximation, obtainable by expanding equation (4.4) of [15], reads

$$
\left\langle q^{2}\right\rangle_{c l}=\frac{\left(k_{B} T\right)}{\omega_{0}^{2}}+\frac{\hbar^{2}}{\left(k_{B} T\right)} \frac{1}{12}-\frac{\hbar^{3}}{\left(k_{B} T\right)^{2}} \frac{\eta \zeta(3)}{4 \pi^{2}}-\frac{\hbar^{4}}{\left(k_{B} T\right)^{3}} \frac{\left(\omega_{0}^{2}-\eta^{2}\right)}{720}+\cdots
$$

Alternatively, one can approach the classical limit of (5.6) by expanding the correlator $F\left(\tau^{\prime}-\tau^{\prime \prime}\right)$ in terms of $\delta\left(\tau^{\prime}-\tau^{\prime \prime}\right)$ and of its derivatives, as outlined in Appendix 3 .

\subsubsection{Low Temperatures}

Near the quantum or zero-temperature limit $\alpha \rightarrow \infty$, an asymptotic approximation follows on substituting into (5.11) from (4.23)b. We call the contribution from absolute zero $\langle\ldots\rangle^{(0)}$, and the leading correction to this $\langle\ldots\rangle^{(1)}$. With $\int_{-\infty}^{\infty} d u \ldots=2 \int_{0}^{\infty} d u \ldots$ and $u^{2}=y$, one finds

$$
\alpha \rightarrow \infty: \quad\left\langle q^{2}\right\rangle \rightarrow\left\langle q^{2}\right\rangle^{(0)}=\frac{\hbar}{2 \omega_{0}} \digamma(g), \quad \digamma(g) \equiv \frac{g}{\pi} \int_{0}^{\infty} d y \frac{1}{\left[y^{2}+\left(-2+g^{2}\right) y+1\right]},
$$

where capital digamma $\digamma(g)$ must not be confused with the correlator $F(\tau)$. Integration yields

$$
0<g^{2}<2: \quad \digamma \equiv \frac{1}{\sqrt{1-g^{2} / 4}}\left\{1-\frac{1}{\pi} \tan ^{-1}\left[\frac{g \sqrt{1-g^{2} / 4}}{1-g^{2} / 2}\right]\right\} .
$$

This tallies with (B.28) in [7], and with (4.7), (4.8) in [15], as can be seen by noting that $\kappa$ and $\sigma$ in [15] are our $g / 2$ and $2 \alpha$, and setting $g=2 \sin (\chi)$ in both expressions. 
The expressions for higher values of $g^{2}$ follow either by analytic continuation or by reevaluating $\int_{0}^{\infty} d y \ldots$ As $g^{2}$ rises through 2 , the arctangent rises through $\pi / 2$, and

$$
2<g^{2}<4: \quad \digamma=\frac{1}{\sqrt{1-g^{2} / 4}}\left\{\frac{1}{\pi} \tan ^{-1}\left[\frac{g \sqrt{1-g^{2} / 4}}{g^{2} / 2-1}\right]\right\} .
$$

For $g^{2}>4$ the oscillator is overdamped, in that (4.14) indicates pure imaginary $\Omega_{0} \equiv$ $\sqrt{\omega_{0}^{2}-\eta^{2} / 4}=i \omega_{0} \sqrt{g^{2} / 4-1}$, replacing the arctangent with a logarithm. By continuity

$$
\begin{aligned}
4<g^{2}: \quad \digamma & =\frac{1}{\sqrt{g^{2} / 4-1}} \frac{1}{\pi} \tanh ^{-1}\left[\frac{g \sqrt{g^{2} / 4-1}}{g^{2} / 2-1}\right] \\
& =\frac{1}{\sqrt{g^{2} / 4-1}} \frac{1}{2 \pi} \log \left[\frac{g^{2} / 2-1+g \sqrt{g^{2} / 4-1}}{g^{2} / 2-1-g \sqrt{g^{2} / 4-1}}\right] .
\end{aligned}
$$

In (5.14-5.16) arctan, arctanh, and log all signify the principal branch.

As $g$ rises, $\digamma$ falls monotonically from 1 to 0 ; the quickest way to see this is to plot it successively over the three ranges in question. Analytically,

$$
\frac{\partial \digamma}{\partial g}=\frac{1}{1-g^{2} / 4}\left[\frac{g \digamma}{4}-\frac{1}{\pi}\right]
$$

and

$$
\begin{aligned}
\digamma\left(g^{2} \ll 1\right)= & 1-g / \pi+g^{2} / 8+\cdots, \quad \digamma\left(g^{2}=2\right)=1 / \sqrt{2}, \quad \digamma\left(g^{2}=4\right)=2 / \pi \\
\digamma\left(g^{2} \gg 1\right)= & (1 / \pi) \log (g)\left[4 / g+8 / g^{3}-24 / g^{5}+\cdots\right] \\
& -(1 / \pi)\left[4 / g^{3}+14 / g^{5}+\cdots\right] .
\end{aligned}
$$

At nonzero but very low $T$ the corrections to $\left\langle q^{2}\right\rangle^{(0)}$ come from the exponentials in (4.23)b, with $x \rightarrow 2 \alpha n u$. As $\alpha \rightarrow \infty$ the integrand is dominated by small $u$; therefore we expand

$$
1 / D^{2}=\sum_{m=0}^{\infty} c_{m} u^{2 m}, \quad c_{0}=1,
$$

and invert the order of the summations:

$$
\begin{aligned}
\mathcal{K}-\mathcal{K}^{(0)} & =\sum_{n=1}^{\infty} \mathcal{K}^{(n)}=\frac{g}{2 \pi} 2 \sum_{m=0}^{\infty} c_{m} 2 \sum_{n=1}^{\infty} \int_{0}^{\infty} d u u^{2 m+1} \exp (-2 \alpha u n) \\
& =\frac{2 g}{\pi} \sum_{m=0}^{\infty} c_{m} \frac{(2 m+1) !}{(2 \alpha)^{2 m+2}} \sum_{n=1}^{\infty} \frac{1}{n^{2 m+2}} \\
& =\frac{2 g}{\pi} \sum_{m=0}^{\infty} c_{m} \frac{(2 m+1) !}{\left(\hbar \omega_{0} / k_{B} T\right)^{2 m+2}} \zeta(2 m+2) .
\end{aligned}
$$

We keep only the first term, with $m=0$ and $\zeta(2)=\pi^{2} / 6$. Then

$$
\mathcal{K}^{(1)} \simeq \frac{g \pi}{3}\left(\frac{k_{B} T}{\hbar \omega_{0}}\right)^{2} \Rightarrow\left\langle q^{2}\right\rangle^{(1)} \simeq \frac{g \pi}{3} \frac{\left(k_{B} T\right)^{2}}{\hbar \omega_{0}^{3}} .
$$

This tallies with the second term on the right of Eq. (4.7) in [15]. 


\section{Part II: Coupled Oscillators}

\section{Introductory}

\subsection{Generalities}

We consider two dynamically identical oscillators linearly coupled to each other, and identically coupled each to its own thermostat. Write $H_{0}$ for their Hamiltonian and $X, \xi$ for their normal modes in absence of the thermostats:

$$
\begin{aligned}
H_{0} & =H_{1,0}+H_{2,0}+V=(1 / 2)\left[\dot{q}_{1}^{2}+\dot{q}_{2}^{2}\right]+\left(\omega_{0}^{2} / 2\right)\left[q_{1}^{2}+q_{2}^{2}+2 \lambda q_{1} q_{2}\right] \\
V & =\lambda \omega_{0}^{2} q_{1} q_{2}, \quad \omega_{p, n}^{2} \equiv \omega_{0}^{2}(1 \pm \lambda), \quad \text { stability } \Rightarrow|\lambda|<1 \\
X & =\left(q_{1}+q_{2}\right) / 2, \quad \xi=q_{1}-q_{2}, \quad q_{1}=X+\xi / 2, \quad q_{2}=X-\xi / 2 \\
H_{0} & =\left[\dot{X}^{2}+\omega_{p}^{2} X^{2}\right]+\left[\dot{\xi}^{2}+\omega_{n}^{2} \xi^{2}\right] / 4 \equiv H_{p}+H_{n} .
\end{aligned}
$$

The subscripts $p, n$ (for positive and negative) replace the subscripts \pm used in $\mathrm{I}$, in order to avoid confusion with the roots $\omega_{ \pm}=\omega_{0} u_{ \pm}$of the quartic $D^{2}$ from Appendix 1. Perturbation theory in $V$, ie weak-coupling approximations, would expand by powers of $\lambda$.

The oscillators have the same friction constant, and experience mutually uncorrelated Langevin forces $f_{1,2}$ appropriate to temperatures $T_{1,2}$ respectively:

$$
\begin{aligned}
\ddot{q}_{1}+\eta \dot{q}_{1}+\omega_{0}^{2} q_{1}+\lambda \omega_{0}^{2} q_{2} & =f_{1,} \quad \ddot{q}_{2}+\eta \dot{q}_{2}+\omega_{0}^{2} q_{2}+\lambda \omega_{0}^{2} q_{1}=f_{2}, \\
\left\langle f_{1,2}\left(t^{\prime}\right) f_{1,2}\left(t^{\prime \prime}\right)\right\rangle & =F_{1,2}(\tau), \quad\left\langle f_{1}\left(t^{\prime}\right) f_{2}\left(t^{\prime \prime}\right)\right\rangle=0, \quad \tau \equiv t^{\prime}-t^{\prime \prime},
\end{aligned}
$$

where $F_{1,2}(\tau)$ are given by the correlator $(4.21,4.22)$ with $T \rightarrow T_{1,2}$ respectively. We define

$$
\Phi=\left(f_{1}+f_{2}\right) / 2, \quad \varphi=\left(f_{1}-f_{2}\right), \quad f_{1}=\Phi+\varphi / 2, \quad f_{2}=\Phi-\varphi / 2,
$$

so that

$$
\begin{aligned}
\left\langle\Phi\left(t^{\prime}\right) \Phi\left(t^{\prime \prime}\right)\right\rangle & =\left[F_{1}(\tau)+F_{2}(\tau)\right] / 4, \quad\left\langle\varphi\left(t^{\prime}\right) \varphi\left(t^{\prime \prime}\right)\right\rangle=F_{1}(\tau)+F_{2}(\tau), \\
\left\langle\Phi\left(t^{\prime}\right) \varphi\left(t^{\prime \prime}\right)\right\rangle & =\left[F_{1}(\tau)-F_{2}(\tau)\right] / 2 .
\end{aligned}
$$

Then

$$
\ddot{X}+\eta \dot{X}+\omega_{p}^{2} X=\Phi, \quad \ddot{\xi}+\eta \dot{\xi}+\omega_{n}^{2} \xi=\varphi .
$$

Thus, while $X$ and $\xi$ evolve independently of each other as regards their dynamics (witness (6.10)), their statistics are correlated ${ }^{3}$ because (6.9) shows that their drivers $\Phi$ and $\varphi$ are correlated unless $T_{1}=T_{2}$.

On the pattern of (4.12-4.14), the eigenfrequencies of the unforced normal modes are $-i \eta / 2 \pm \Omega_{p, n}$, with

$$
\Omega_{p, n}=\sqrt{\omega_{p, n}^{2}-\eta^{2} / 4}, \quad \Omega_{p, n}^{2}+\eta^{2} / 4=\omega_{p, n}^{2},
$$

written, for simplicity, appropriately to under-damping (even for $\xi$ ). The corresponding Green's functions read

$$
\mathcal{G}_{p, n}(\tau)=\theta(\tau) \frac{1}{\Omega_{p, n}} \exp (-\eta \tau / 2) \sin \left(\Omega_{p, n} \tau\right) .
$$

3 Dorofeyev [11] has written down the quantum Langevin equations for a two-bath model not unlike ours, but is concerned mainly with numerical illustrations of interaction energies rather than with heat flow. 
For instance, on the pattern of (5.1),

$$
(X(t), \xi(t))=\int_{0}^{t} d t^{\prime} \mathcal{G}_{p, n}\left(t, t^{\prime}\right)\left(\Phi\left(t^{\prime}\right), \varphi\left(t^{\prime}\right)\right)=\int_{0}^{t} d \tau \mathcal{G}_{p, n}(\tau)(\Phi(t-\tau), \varphi(t-\tau)) .
$$

Finally, recalling the scaled parameters $g=\eta / \omega_{0}, \alpha=\hbar \omega_{0} / 2 k_{B} T$, and $u=\omega / \omega_{0}$, and in view of (6.2), we define

$$
\alpha_{p, n} \equiv \alpha \sqrt{1 \pm \lambda}, \quad g_{p, n} \equiv g / \sqrt{1 \pm \lambda}, \quad\left(u_{p, n}, \bar{u}_{p, n},\right)=(u, \bar{u}) / \sqrt{1 \pm \lambda},
$$

where $\bar{u}$ and $\bar{u}_{p, n}$ anticipate auxiliary cutoffs that will be needed in Sect. 7.1.

The heat-flow $\mathcal{P}$ from 1 to 2 is the mean rate at which work is done on $q_{2}$ by the force $-\partial V / \partial q_{2}=-\lambda \omega_{0}^{2} q_{1}$ exerted on it by oscillator 1 :

$$
\mathcal{P}=-\lambda \omega_{0}^{2}\left\langle q_{1} \dot{q}_{2}\right\rangle=-\lambda \omega_{0}^{2}\langle\dot{X} \xi\rangle=\lambda \omega_{0}^{2}\langle X \dot{\xi}\rangle .
$$

Clearly $\mathcal{P}$ and therefore $\langle X \dot{\xi}\rangle$ must have the sign of $T_{1}-T_{2}$, and cannot depend on the sign of $\lambda$.

\subsection{The Classical Limit}

The classical limits of $\left\langle X^{2}\right\rangle,\left\langle\xi^{2}\right\rangle,\langle X \xi\rangle$, and $\langle X \dot{\xi}\rangle$ were found in [3], and will be re-derived in Sect. 7.1. In our present notation, and in terms of $\Delta T \equiv T_{1}-T_{2}$, of $\bar{T} \equiv\left(T_{1}+T_{2}\right) / 2$, and of $T_{1,2}=\bar{T} \pm \Delta T / 2$, they are

$$
\left[\begin{array}{ll}
\langle X X\rangle_{c l}, & \langle X \xi\rangle_{c l} \\
\langle\xi X\rangle_{c l}, & \langle\xi \xi\rangle_{c l}
\end{array}\right]=\frac{k_{B}}{2 \omega_{0}^{2}}\left[\begin{array}{ll}
\bar{T} /(1+\lambda), & \Delta T g^{2} /\left(g^{2}+\lambda^{2}\right) \\
\Delta T g^{2} /\left(g^{2}+\lambda^{2}\right), & 4 \bar{T} /(1-\lambda)
\end{array}\right],
$$

where the diagonal elements depend neither on $g$ nor on $\Delta T$; and

$$
\langle X \dot{\xi}\rangle_{c l}=-\langle\dot{X} \xi\rangle_{c l}=\frac{k_{B} \Delta T}{2 \omega_{0}} \cdot \frac{g \lambda}{\left(g^{2}+\lambda^{2}\right)} \Rightarrow \mathcal{P}_{c l}=\frac{k_{B} \Delta T \omega_{0}}{2} \cdot \frac{g \lambda^{2}}{\left(g^{2}+\lambda^{2}\right)} .
$$

The limits (6.16) suffice to show that in this system there is no local thermal equilibrium (no LTE). In other words the states of the individual oscillators differ from what they would be if the oscillators were decoupled, with each in equilibrium at the temperature of its thermostat. For instance, $L T E$ would replace the exact result

$$
\left\langle q_{1}^{2}\right\rangle_{c l}=\left\langle\left(X+\frac{\xi}{2}\right)^{2}\right\rangle_{c l}=\left\langle X^{2}+\frac{\xi^{2}}{4}+X \xi\right\rangle_{c l}=\frac{k_{B}\left\{T_{1} 2 \eta^{2}+T_{2} \lambda^{2}\left(\omega_{0}^{2}+\eta^{2}\right)\right\}}{2 \omega_{0}^{2}\left(1-\lambda^{2}\right)\left(\eta^{2}+\lambda^{2} \omega_{0}^{2}\right)}
$$

with the weak-coupling approximation

$$
\left\langle q_{1}^{2}\right\rangle_{L T E, c l}=\left\langle q^{2}\right\rangle_{c l}=k_{B} T_{1} / \omega_{0}^{2}
$$

namely with the classical limit of (5.12), appropriate to a single isolated oscillator at $T_{1}$. Correspondingly, for the classical heat flow LTE would yield the weak-coupling limit of (6.17)b, namely

$$
\mathcal{P}_{L T E, c l}=\lambda^{2} \omega_{0} k_{B} \Delta T / 2 g .
$$

The direct calculation is outlined in Sect. 7.2.2. Specifically, in our system perturbative approximations require not only $\lambda \ll 1$, but also $\lambda^{2} \ll g^{2}$. This is a far more restrictive 
condition, because, as Sect. 8.1 will suggest, in atomic and condensed-state physics damping is usually weak, with $g^{2} \ll 1$. By contrast, large $g$ and overdamping are common in circuitry.

Ciliberto et al. [8] have measured the energy flow between two electrically coupled resistors, under conditions which in our terms correspond to high temperatures and to overdamping with $g^{2} \gg \lambda^{2}$. In the weak-coupling limit $g \lambda^{2} /\left(g^{2}+\lambda^{2}\right) \rightarrow \lambda^{2} / g$ our (6.16) and (6.17)a tally with equations (3)-(6) near the end of their paper, and our $\mathcal{P}_{c l}$ reduces to their Eq. (7), confirmed within errors by their measurements. (See also Ciliberto et al. [9].) Elaborate analyses of the density matrix in the high-temperature and weak-coupling limits, with many numerical examples, are given by Ghesquière et al. [14], whose Appendix B specifies hightemperature expectation values tallying precisely with our (6.16) and (6.17)a. [An earlier paper (Ghesquière et al. [13]) stresses that there are combinations of input parameters for which no steady state is ever reached, but appears not to have traced this to the manifest instability of the Hamiltonian ensuing from $|\lambda|>1$, as noted in our Eq. (6.2).]

To conclude, we anticipate Sect. 7.2 and parts of Appendix 4 to stress that, in quantum mechanics as well as in its classical limit, it is the general rule that LTE and the fluctuationdissipation theorem appropriate to LTE apply only under the assumptions of linear response theory, i.e. only to leading order of perturbation theory. Regarding quantum friction between a neutral atom and a half-space this is amply illustrated by Intravaia et al. [16]; and the present writer suspects that eventually it might prove worth while to take a similar look at the validity limits of $L T E$ as applied to nonequilibrium steady-state Casimir forces in much of the recent literature (see e.g. Domingues et al. [10], Messina and Antezza [22], Krüger et al. [18], Bimonte [4], and their references). For instance, prima facie it is inconsistent to assume $L T E$ and yet calculate forces to higher than leading order in the coupling. Some of the difficulties met beyond LTE are apparent from the review by Dubi and Di Ventra [12].

\section{The Correlator $\langle X \dot{\xi}\rangle$}

This is the expectation value that governs the heat flow $\mathcal{P}=-\lambda \omega_{0}^{2}\left\langle q_{1} \dot{q}_{2}\right\rangle=\lambda \omega_{0}^{2}\langle X \dot{\xi}\rangle$, and calculating it exactly requires an irreducible minimum of regrettably tedious detail. Indeed it is fortunate, and noteworthy, that the underlying frequency-integrals $\int_{-\infty}^{\infty} d \omega \ldots$ converge; the more so because (unless the classical limit is taken right at the outset) those needed for $\left\langle\dot{q}^{2}\right\rangle$ and for $\left\langle\dot{X}^{2}, \dot{\xi}^{2}, \dot{X} \dot{\xi}\right\rangle$ already require cutoffs $|\omega|<\omega_{D}$ on the frequencies of the thermostat (see e.g. [15]), such as might be motivated in our model by a Debye-type appeal to the granularity of the string. The main results, for readers willing to take them on trust, are (7.10-7.12) for arbitrary temperatures, (7.14) for high $T$, and (7.16) plus (7.20) for low $T$.

It will prove convenient to define, acting on any function $w$ of temperature, the operation

$$
\Delta_{T} w(T) \equiv w\left(T_{1}\right)-w\left(T_{2}\right) .
$$

For instance, (6.9) can be written as $\left\langle\Phi\left(t^{\prime}\right) \varphi\left(t^{\prime \prime}\right\rangle=\Delta_{T} F(\tau) / 2\right.$.

\subsection{Exact Calculation}

From

$$
X(t)=\int_{0}^{t} d \tau^{\prime} \mathcal{G}_{p}\left(\tau^{\prime}\right) \Phi\left(t-\tau^{\prime}\right), \quad \dot{\xi}(t)=\int_{0}^{t} d \tau^{\prime \prime} \frac{\partial \mathcal{G}_{n}\left(\tau^{\prime \prime}\right)}{\partial \tau^{\prime \prime}} \varphi\left(t-\tau^{\prime \prime}\right),
$$


plus invariance under time translation and time reversal, we find

$$
\begin{gathered}
\langle X \dot{\xi}\rangle=\int_{0}^{\infty} \int_{0}^{\infty} d \tau^{\prime} d \tau^{\prime \prime} \mathcal{G}_{p}\left(\tau^{\prime}\right) \frac{\partial \mathcal{G}_{n}\left(\tau^{\prime \prime}\right)}{\partial \tau^{\prime \prime}}\left\langle\Phi\left(\tau^{\prime}\right) \varphi\left(\tau^{\prime \prime}\right)\right\rangle \\
=\frac{1}{2} \int_{0}^{\infty} \int_{0}^{\infty} d \tau^{\prime} d \tau^{\prime \prime} \mathcal{G}_{p}\left(\tau^{\prime}\right) \frac{\partial \mathcal{G}_{n}\left(\tau^{\prime \prime}\right)}{\partial \tau^{\prime \prime}}\left\{F_{T_{1}}\left(\tau^{\prime}-\tau^{\prime \prime}\right)-F_{T_{2}}\left(\tau^{\prime}-\tau^{\prime \prime}\right)\right\} .
\end{gathered}
$$

Thus, defining

$$
\langle X \dot{\xi}\rangle_{T} \equiv \frac{1}{2} \int_{0}^{\infty} \int_{0}^{\infty} d \tau^{\prime} d \tau^{\prime \prime} \mathcal{G}_{p}\left(\tau^{\prime}\right) \frac{\partial \mathcal{G}_{n}\left(\tau^{\prime \prime}\right)}{\partial \tau^{\prime \prime}} F_{T}\left(\tau^{\prime}-\tau^{\prime \prime}\right)
$$

one has

$$
\langle X \dot{\xi}\rangle=\langle X \dot{\xi}\rangle_{T_{1}}-\langle X \dot{\xi}\rangle_{T_{2}}=\Delta_{T}\langle X \dot{\xi}\rangle_{T}
$$

Substitution for $F$ from $(4.21,4.22)$ then leads to

$$
\begin{gathered}
\langle X \dot{\xi}\rangle=-\Delta_{T} \frac{\eta \hbar}{4 \pi i} \int_{-\infty}^{\infty} d \omega \omega^{2} \operatorname{coth}(\beta \hbar \omega / 2) \times \\
\times \int_{0}^{\infty} \int_{0}^{\infty} d \tau^{\prime} d \tau^{\prime \prime} \mathcal{G}_{p}\left(\tau^{\prime}\right) \mathcal{G}_{n}\left(\tau^{\prime \prime}\right) \sin \left[\omega\left(\tau^{\prime}-\tau^{\prime \prime}\right)\right] .
\end{gathered}
$$

Under $\int_{-\infty}^{\infty} d \omega \ldots$ we can replace $\sin \left[\omega\left(\tau^{\prime}-\tau^{\prime \prime}\right)\right] \rightarrow \exp \left[i \omega\left(\tau^{\prime}-\tau^{\prime \prime}\right)\right] / i$ :

$$
\begin{aligned}
\langle X \dot{\xi}\rangle= & -\Delta_{T} \frac{\eta \hbar}{4 \pi i} \int_{-\infty}^{\infty} d \omega \omega^{2} \operatorname{coth}(\beta \hbar \omega / 2) \\
& \times\left[\int_{0}^{\infty} d \tau^{\prime} \mathcal{G}_{p}\left(\tau^{\prime}\right) \exp \left(i \omega \tau^{\prime}\right)\right]\left[\int_{0}^{\infty} d \tau^{\prime \prime} \mathcal{G}_{n}\left(\tau^{\prime \prime}\right) \exp \left(-i \omega \tau^{\prime}\right)\right] .
\end{aligned}
$$

Using (6.12) to evaluate the time-integrals, and rationalizing, one obtains

$$
\langle X \dot{\xi}\rangle=-\Delta_{T} \frac{\eta \hbar}{4 \pi i} \int_{-\infty}^{\infty} d \omega \omega^{2} \operatorname{coth}(\beta \hbar \omega / 2) \frac{\left[\omega_{p}^{2}-\omega^{2}+i \omega \eta\right]}{\left[\left(\omega_{p}^{2}-\omega^{2}\right)^{2}+\eta^{2} \omega^{2}\right]} \frac{\left[\omega_{n}^{2}-\omega^{2}-i \omega \eta\right]}{\left[\left(\omega_{n}^{2}-\omega^{2}\right)^{2}+\eta^{2} \omega^{2}\right]} .
$$

Since only the imaginary part of the numerator survives under $\int_{-\infty}^{\infty} d \omega \ldots$, change $\int_{-\infty}^{\infty} d \omega \ldots \rightarrow 2 \int_{0}^{\infty} d \omega \ldots$, note $-\omega_{p}^{2}+\omega_{n}^{2}=-2 \lambda \omega_{0}^{2}$, set $\omega / \omega_{0}=u$ and $u^{2}=y$, recall $\alpha \equiv \beta \hbar \omega / 2$, and find

$$
\begin{gathered}
\langle X \dot{\xi}\rangle=\Delta_{T} \frac{g^{2} \hbar}{\pi} \int_{0}^{\infty} d u \operatorname{coth}(\alpha u) \frac{\lambda u^{3}}{\left[\left(1+\lambda-u^{2}\right)^{2}+g^{2} u^{2}\right]\left[\left(1-\lambda-u^{2}\right)^{2}+g^{2} u^{2}\right]}, \\
\langle X \dot{\xi}\rangle=\Delta_{T} \frac{g^{2} \hbar}{2 \pi} \int_{0}^{\infty} d y \operatorname{coth}(\alpha \sqrt{y}) \frac{\lambda y}{\left[(1+\lambda-y)^{2}+g^{2} y\right]\left[(1-\lambda-y)^{2}+g^{2} y\right]}
\end{gathered}
$$


To deal with (7.8) or with (7.9) we separate the integrands into partial fractions, splitting each integral into two components which, individually, diverge. On recombination the divergences cancel; meanwhile we handle them by imposing purely auxiliary cutoffs ${ }^{4}|u| \leq \bar{u} \Rightarrow$ $y \leq \bar{y} \equiv \bar{u}^{2}$, ie $|\omega| \leq \bar{\omega} \equiv \omega_{0} \bar{u}$, on the understanding that $\bar{u} \gg(\lambda, g)$, and implement $\bar{u} \rightarrow \infty$ as soon as possible. We shall telescope this prescription into $\bar{u} \rightarrow \infty$ without further comment. In this way we find

$$
\begin{gathered}
\langle X \dot{\xi}\rangle=\langle X \dot{\xi}\rangle_{p}-\langle X \dot{\xi}\rangle_{n}, \quad\langle X \dot{\xi}\rangle_{n}=\left[\langle X \dot{\xi}\rangle_{p} \text { with } \lambda \rightarrow-\lambda\right], \\
\langle X \dot{\xi}\rangle_{p}=-\Delta_{T} \frac{\hbar g^{2}}{4 \pi\left(g^{2}+\lambda^{2}\right)} \int_{0}^{\bar{u}_{p}} d u \operatorname{coth}(\alpha u) \frac{u\left[u^{2}-(1+\lambda)^{2}\right]}{\left[\left(1+\lambda-u^{2}\right)^{2}+g^{2} u^{2}\right]},
\end{gathered}
$$

where subscripts $p, n$ specify not temperature but the sign of $\lambda$, and $\bar{u}_{p}$ etc are as defined in (6.14). Scaling simplifies this to

$$
\langle X \dot{\xi}\rangle_{p}=-\Delta_{T} \frac{\hbar g^{2}}{4 \pi\left(g^{2}+\lambda^{2}\right)} \int_{0}^{\bar{u}_{p}} d u_{p} \operatorname{coth}\left(\alpha_{p} u_{p}\right) \frac{u_{p}\left[u_{p}^{2}-(1+\lambda)\right]}{D^{2}\left(u_{p}, g_{p}\right)} .
$$

Near-classically, $\operatorname{coth}\left(\alpha_{p} u_{p}\right) \simeq 1 / \alpha_{p} u_{p}+\alpha_{p} u_{p} / 3$ produces

$$
\left[\int_{0}^{\bar{u}} d u_{p} \ldots\right]_{c l} \simeq \frac{1}{\alpha_{p}} \int_{0}^{\infty} d u_{p} \frac{\left[u_{p}^{2}-(1+\lambda)\right]}{D^{2}\left(u_{p}, g_{p}\right)}+\frac{\alpha_{p}}{3} \int_{0}^{\bar{u}_{p}} d u_{p} \frac{u_{p}^{4}-(1+\lambda) u_{p}^{2}}{D^{2}\left(u_{p}, g_{p}\right)} .
$$

The integrals are given in Appendix 1. On combination with $\langle X \dot{\xi}\rangle_{n, c l}$ the divergences cancel, and the end-result reads

$$
\langle X \dot{\xi}\rangle_{c l} \simeq \Delta_{T} \frac{g \lambda}{\left(\lambda^{2}+g^{2}\right)}\left\{\frac{k_{B} T}{2 \omega_{0}}+\frac{\hbar^{2} \omega_{0}}{24 k_{B} T}\right\} .
$$

The first term tallies with (6.17) as it should. The second term could have been found more quickly by the method of Appendix 3.

When both oscillators are thermostatted to absolute zero, the prefactor $\Delta_{T}$ causes $\langle X \dot{\xi}\rangle$ to vanish; but the end-results at finite temperatures do feature the zero-temperature limits of $\langle X \dot{\xi}\rangle_{T_{1,2}}$ individually. To avoid overcrowding the notation, we now switch as in Sect. 5.2.3 to indicating $T=0$ by superscripts (0) instead of subscripts 0 : for instance, $\langle X \dot{\xi}\rangle_{T=0}$ from (7.5) now becomes $\langle X \dot{\xi}\rangle^{(0)}$. In (7.9) we then replace coth $(\alpha \sqrt{y}) \ldots \rightarrow 1$, and find

$$
\begin{aligned}
\left\langle\left. X \dot{\xi}\right|_{p} ^{(0)}\right. & =-\frac{\hbar g^{2}}{8 \pi\left(g^{2}+\lambda^{2}\right)} \int_{0}^{\bar{y}_{p}} d y_{p} \frac{\left[y_{p}-(1+\lambda)\right]}{\left(1-y_{p}\right)^{2}+g_{p}^{2} y_{p}}= \\
& =\frac{\hbar g^{2}}{8 \pi\left(g^{2}+\lambda^{2}\right)} \frac{1}{2}\left\{-\log (\bar{y})+\log (1+\lambda)+\left[\frac{\lambda}{g_{p}}+\frac{g_{p}}{2}\right] \pi \digamma\left(g_{p}\right)\right\} .
\end{aligned}
$$

\footnotetext{
4 These cutoffs have no physical significance. In particular they have nothing in common with the Debye cutoffs $\omega_{D}$ needed to calculate $\left\langle\dot{q}^{2}\right\rangle$, which have, as was pointed out in the preamble to this section.
} 
The first term emerges on reconverting from $\bar{y}_{p}$ to $\bar{y}$, in order to explicate the cancellation of the divergence from the difference (7.10)a. The end-result reads

$$
\begin{aligned}
\langle X \dot{\xi}\rangle^{(0)}= & \frac{\hbar g^{2}}{8 \pi\left(g^{2}+\lambda^{2}\right)}\left\{\log \left(\frac{1+\lambda}{1-\lambda}\right)+\right. \\
& \left.+\frac{\pi \lambda}{g}\left[\digamma\left(g_{p}\right)+\digamma\left(g_{n}\right)\right]+\frac{1}{2}\left[g_{p}^{2} \digamma\left(g_{p}\right)-g_{n}^{2} \digamma\left(g_{n}\right)\right]\right\} .
\end{aligned}
$$

We observe (i) that (7.16) vanishes with $\lambda$, as it must; and (ii) that to leading order it reads

$$
\langle X \dot{\xi}\rangle^{(0)}=-\frac{\lambda \hbar g}{4} \frac{\partial}{\partial g}\left[\frac{\digamma(g)}{g}\right]+\mathcal{O}\left(\lambda^{2}\right) .
$$

The finite-temperature corrections $\langle X \dot{\xi}\rangle^{(m)}$ follow by the direct method already used for the $\left\langle q^{2}\right\rangle^{(m)}$ in Sect. 5.2.3: in view of

$$
\frac{u^{2}}{\left[\left(1+\lambda-u^{2}\right)^{2}+g^{2} u^{2}\right]\left[\left(1-\lambda-u^{2}\right)^{2}+g^{2} u^{2}\right]}=\sum_{m=1}^{\infty} c_{m} u^{2 m}, \quad c_{1}=\frac{1}{\left(1-\lambda^{2}\right)^{2}}
$$

one finds

$$
\begin{gathered}
\langle X \dot{\xi}\rangle^{(m)}=\Delta_{T} \frac{2 \lambda \hbar g^{2}}{\pi} \sum_{m=1}^{\infty} c_{m}(2 m+1) ! \zeta(2 m+2)\left(\frac{k_{B} T}{\hbar \omega_{0}}\right)^{2 m+2} \\
\langle X \dot{\xi}\rangle^{(1)}=\Delta_{T} \frac{\lambda g^{2}}{\left(1-\lambda^{2}\right)^{2}} \frac{2 \pi^{3}}{15}\left(\frac{k_{B} T}{\hbar \omega_{0}}\right)^{4} .
\end{gathered}
$$

\subsection{Linear Response}

\subsubsection{Pertubation Theory}

In spite of the limitations of linear-response theory outlined in Sect. 6, we sketch its application to the correlator $\langle X \dot{\xi}\rangle$, partly because the approximation is so widespread in the literature, but mainly because splitting the exact results into $p$ and $n$ parts as in (7.10-7.12) has produced expressions so awkward that the writer thought them much in need of the simple checks available to leading order in $\lambda$. One should bear in mind that approximations to this order, now identified by primes, are not applicable to weak-dissipation regimes where $g \lesssim \lambda$.

For $\langle X \dot{\xi}\rangle^{\prime}$, Eq. (7.8) yields

$$
\begin{gathered}
\langle X \dot{\xi}\rangle^{\prime}=\Delta_{T} \frac{\lambda g^{2} \hbar}{\pi} \int_{0}^{\infty} d u \operatorname{coth}(\alpha u) \frac{u^{3}}{\left[D^{2}(u, g)\right]^{2}} \\
=-\Delta_{T} \frac{\lambda g^{2} \hbar}{\pi} \frac{\partial}{\partial g^{2}} \int_{0}^{\infty} d u \operatorname{coth}(\alpha u) \frac{u}{D^{2}(u, g)}=-\frac{1}{2} \lambda \omega_{0} \Delta_{T} g \frac{\partial}{\partial g}\left(\frac{\left\langle q^{2}\right\rangle}{g}\right),
\end{gathered}
$$

where the wholly unexpected rightmost expression follows by comparison with (5.10). 
Classically (7.22) reduces to

$$
\langle X \dot{\xi}\rangle_{c l}^{\prime}=\frac{\lambda k_{B} \Delta T}{2 \omega_{0} g}
$$

tallying with (6.17)a. At absolute zero, (7.21), with $y \equiv u^{2}$, reduces to

$$
\begin{gathered}
\left\langle\left. X \dot{\xi}\right|^{(0)^{\prime}}=\frac{\lambda g^{2} \hbar}{2 \pi} \int_{0}^{\infty} d y \frac{y}{\left[(1-y)^{2}+g^{2} y\right]^{2}}\right. \\
=-\frac{\lambda g \hbar}{4 \pi} \frac{\partial}{\partial g} \int_{0}^{\infty} d y \frac{1}{\left[(1-y)^{2}+g^{2} y\right]}=-\frac{\lambda \hbar g}{4} \frac{\partial}{\partial g}\left[\frac{\digamma(g)}{g}\right],
\end{gathered}
$$

tallying with (7.17).

\subsubsection{Local Thermal Equilibrium}

As promised in Sect. 6, we derive the classical weak-coupling result (7.23) directly from the assumption of local thermal equilibrium ( $L T E$ ).

Let $q_{1,2}^{(0)}(t)$ be the exact solutions, taken from Sect. 5, for isolated oscillators at temperatures $T_{1,2}$ respectively. The exact equations of motion (6.5) of the coupled system entail

$$
q_{1}(t)=\int_{0}^{t} d t^{\prime} \mathcal{G}\left(t, t^{\prime}\right)\left[f_{1}\left(t^{\prime}\right)-\lambda \omega_{0}^{2} q_{2}\left(t^{\prime}\right)\right]=q_{1}^{(0)}(t)-\lambda \omega_{0}^{2} \int_{0}^{t} d t^{\prime} \mathcal{G}\left(t, t^{\prime}\right) q_{2}\left(t^{\prime}\right),
$$

$$
\dot{q}_{2}(t)=\int_{0}^{t} d t^{\prime \prime} \frac{\partial \mathcal{G}\left(t, t^{\prime \prime}\right)}{\partial t}\left[f_{1}\left(t^{\prime \prime}\right)-\lambda \omega_{0}^{2} q_{2}\left(t^{\prime \prime}\right)\right]=\dot{q}_{2}^{(0)}(t)-\lambda \omega_{0}^{2} \int_{0}^{t} d t^{\prime \prime} \frac{\partial \mathcal{G}\left(t, t^{\prime \prime}\right)}{\partial t} q_{1}\left(t^{\prime \prime}\right) .
$$

LTE replaces $q_{1,2} \rightarrow q_{1,2}^{(0)}$ under the integrals. Then (i) we note that $\left\langle q_{1}^{(0)}\left(t^{\prime}\right) \dot{q}_{2}^{(0)}\left(t^{\prime \prime}\right)\right\rangle=$ $\left\langle q_{1}^{(0)}\left(t^{\prime}\right) f_{2}\left(t^{\prime \prime}\right)\right\rangle=0$, etc; (ii) implement the steady-state limit $t \rightarrow \infty$ analogously to Sect. 5.2.1 for $\left\langle q^{2}\right\rangle$; (iii) integrate by parts with respect to $t^{\prime \prime}$; and find

$$
\left\langle q_{1} \dot{q}_{2}\right\rangle_{L T E}=-\lambda \omega_{0}^{2} \int_{0}^{\infty} d \tau \mathcal{G}(\tau)\left\langle q_{2}^{(0)}(0) \dot{q}_{2}^{(0)}(\tau)-\dot{q}_{1}^{(0)}(\tau) q_{1}^{(0)}(0)\right\rangle .
$$

This applies at arbitrary temperatures. But classically the order in which $q_{2}^{(0)}(0)$ and $\dot{q}_{2}^{(0)}(\tau)$ enter is irrelevant, whence

$$
\left\langle q_{1} \dot{q}_{2}\right\rangle_{L T E, c l}=\lambda \omega_{0}^{2} \Delta_{T} \int_{0}^{\infty} d \tau \mathcal{G}(\tau) \dot{J}_{c l}(\tau), \quad J_{c l}(\tau) \equiv\left\langle q^{(0)}(\tau) q^{(0)}(0)\right\rangle_{c l},
$$

with $J$ the position correlator of an isolated Ohmically damped harmonic oscillator. Remarkably, in the classical limit, best implemented as $\hbar \rightarrow 0$, Eq. (3.5) of [15] yields

$$
J_{c l}(\tau)=\frac{k_{B} \tau}{\omega_{0}^{2} \Omega_{0}} \exp \left(-\frac{\eta \tau}{2}\right)\left[\Omega_{0} \cos \left(\Omega_{0} \tau\right)+\frac{\eta}{2} \sin \left(\Omega_{0} \tau\right)\right], \quad \dot{J}_{c l}(\tau)=-k_{B} \tau \mathcal{G}(\tau) .
$$


Finally, substituting (7.29)b into (7.28)a, we obtain

$$
\begin{gathered}
\left\langle q_{1} \dot{q}_{2}\right\rangle_{L T E, c l}=-\lambda \omega_{0}^{2} k_{B} \Delta_{T} \int_{0}^{\infty} d \tau \mathcal{G}^{2}(\tau)=-\frac{\lambda k_{B} \Delta T}{2 \eta}=-\frac{\lambda k_{B} \Delta T}{2 \omega_{0} g} \Rightarrow \\
\mathcal{P}_{L T E, c l}=-\lambda \omega_{0}^{2}\left\langle q_{1} \dot{q}_{2}\right\rangle_{L T E, c l}=\frac{\lambda^{2} \omega_{0} k_{B} \Delta T}{2 g} .
\end{gathered}
$$

\section{The Heat-Flow $\mathcal{P}$}

By (6.15) the heat flow per unit time is $\mathcal{P}=\lambda \omega_{0}^{2}\langle X \dot{\xi}\rangle$. We focus on oscillators mimicking atoms or molecules, rather than on coupled circuits like those referenced near the end of Sect. 6. Section 8.1 considers plausible values of the crucial input parameters; Sect. 8.2 their bearing on our approximations; and Sect. 8.3 the way $\mathcal{P}$ is likely to vary with temperature and with distance. Closed expressions for $\mathcal{P}$ emerge only when $T_{1}$ and $T_{2}$ are both low, both high, or one high and one low.

\subsection{Parameter Values}

\subsubsection{Atomic Units}

We form rough estimates in atomic units (au), where

$$
\begin{gathered}
\hbar=|e|=\left(m_{e}=\text { electron mass }\right)=\left(a_{B}=\text { Bohr radius }\right)=1, \\
e^{2} / \hbar c \simeq 1 / 137, \quad c \simeq 137 .
\end{gathered}
$$

The orders of magnitude of the oscillator parameters are chosen so as to mimick atoms or nonpolar molecules, except in Appendix 4, where they will mimick the surface modes of macroscopic half-spaces of Drude-modelled insulators or conductors. Thus, if $\omega_{0}$ is governed by electronic transitions, i.e. if it is in the optical range, then $\omega_{0}=\omega_{\text {atomic }, 0} \sim 1 \mathrm{au}$. On the other hand, if $\omega_{0}$ is governed by molecular or solid-state transitions, i.e. if it is in the infrared, then it is smaller by a factor of order $\sqrt{m_{e} /(\text { atomic mass })}$, whence $\omega_{\text {molecular }, 0} \sim 10^{-2} \mathrm{au}$. The electrostatic polarizability of the oscillators is of the same order in both scenarios, with $\chi_{0}=e^{2} / m \omega_{0}^{2} \sim a_{B}^{3} \sim \mathcal{O}(1)$.

\subsubsection{Temperatures}

Regarding temperatures the key fact is that 1 au of energy corresponds to $k_{B} T$ with $T \sim 10^{5} \mathrm{~K}$. Hence atomic scenarios with $\omega_{\text {atomic }, 0} \sim 1$ are amply covered by the low- $T$ approximations $\mathcal{P}^{(0)}$ and $\mathcal{P}^{(1)}$ spelled out in Sect. 8.2. By contrast, in molecular or solid-state scenarios we cannot automatically treat room temperatures as either low or high. Consider for instance, anticipating Appendix 4, the remarkable measurements of Ottens et al. [23] on halfspaces near $300 \mathrm{~K}$ : they used sapphire, where the dominant frequencies, which our model collapses into $\omega_{0}$, correspond to temperatures of roughly $600 K$. Then $k_{B} T / \hbar \omega_{0} \sim 1 / 2$, squarely in the range where neither the near-classical nor the low- $T$ approximations to $\langle X \dot{\xi}\rangle$ apply, and only numerical evaluation of the complete expression (6.15) plus (7.10 - 7.12) could serve. 


\subsubsection{Coupling Strength}

The Hamiltonian (6.1) features the coupling term $\lambda q_{1} q_{2} \omega_{0}^{2}$. We identify this with the electrostatic interaction between the oscillator dipole moments $e \tilde{\mathbf{q}}_{1,2}=e \mathbf{q}_{1,2} / \sqrt{m}$, writing their relative position vector (also in atomic units) as $\mathbf{r}$, so that

$$
\lambda=\left(\chi_{0} / r^{3}\right)\left[\hat{\mathbf{q}}_{1} \cdot \hat{\mathbf{q}}_{2}-3\left(\hat{\mathbf{q}}_{1} \cdot \hat{\mathbf{r}}\right)\left(\hat{\mathbf{q}}_{2} \cdot \hat{\mathbf{r}}\right)\right] .
$$

For simplicity we choose $\lambda$ appropriately to dipoles antiparallel to each other and perpendicular to $\mathbf{r}$. Since in atomic units $\chi_{0}$ and $q_{1,2}$ are all of order unity, our estimate of the coupling strengths reads

$$
|\lambda| \sim 1 / r^{3}, \quad(r \text { in } a u) .
$$

One should keep in mind that at distances below or of the order of the oscillator radius $\sim a_{B}$ the dipole-dipole interaction ceases to tell the whole story, and that, with higher multipoles included, $H_{\text {int }}$ is no longer bilinear in $q_{1}$ and $q_{2}$.

\subsubsection{Dissipation}

If $\eta$ in (4.9) stems from Maxwellian radiative reaction in an electronic transition, then

$$
g=\frac{\eta}{\omega_{\text {atomic }, 0}}=\frac{2 e^{2} \omega_{\text {atomic }, 0}}{3 c^{3} m_{e}}=\frac{2}{3}\left(\frac{e^{2}}{\hbar c}\right) \frac{\hbar \omega_{\text {atomic }, 0}}{m_{e} c^{2}} \sim\left(\frac{1}{137}\right)^{3} \sim 4 \times 10^{-7} .
$$

On the other hand (and with direct bearing on Appendix 4), the dispersion relations of solidstate plasmas, mostly with surface-plasmon frequencies $\omega_{S} \sim 1$, would suggest

$$
g_{\text {metals }} \sim \eta / \omega_{S} \sim 10^{-2} \text { to } 10^{-3}, \quad g_{\text {insulators }} \sim \eta / \omega_{S} \sim 10^{-1} \text { to } 10^{-2} .
$$

Accordingly, for applications to atomic or to condensed-state physics,

$$
g \ll 1 .
$$

Note that we are taking $g$ as independent of temperature. This should be at least roughly adequate when $T_{1}$ and $T_{2}$ are both high or both low. Any corrections needed when one is high and one low are likely to be quite laborious.

\subsection{Approximations}

As in (8.7) we consider only $g \ll 1$. Regarding $\lambda$, we note from stability that $|\lambda|<1$, but exclude $1-|\lambda| \ll 1$ for simplicity. We do not assume $|\lambda| \ll 1$, and assume nothing about $\lambda / g$. This suffices to ensure $g_{p, n}=g / \sqrt{1 \pm \lambda} \ll 1$, whence (5.18) entails

$$
\digamma\left(g_{p, n}\right) \simeq 1-g_{p, n} / \pi \text {. }
$$

At low temperatures (7.16) then reduces $\mathcal{P}$ to

$$
\mathcal{P}^{(0)} \simeq \frac{\lambda \hbar g^{2} \omega_{0}^{2}}{8 \pi\left(\lambda^{2}+g^{2}\right)}\left\{\log \left(\frac{1+\lambda}{1-\lambda}\right)+\frac{2 \pi \lambda}{g}-\frac{\lambda}{g}\left[g_{p}^{2}-g_{n}^{2}\right]\right\} \simeq \frac{\lambda^{2} \hbar g \omega_{0}^{2}}{4\left(\lambda^{2}+g^{2}\right)},
$$

while, by (7.20),

$$
\mathcal{P}^{(1)} \simeq \Delta_{T} \frac{\lambda^{2} \hbar g^{2} \omega_{0}^{2}}{\left(1-\lambda^{2}\right)^{2}} \frac{2 \pi^{3}}{15}\left(\frac{k_{B} T}{\hbar \omega_{0}}\right)^{4}
$$


Thus

$$
\mathcal{P}^{(1)} / \mathcal{P}^{(0)} \simeq \frac{g\left(\lambda^{2}+g^{2}\right)}{\left(1-\lambda^{2}\right)^{2}} \frac{8 \pi^{3}}{15}\left(\frac{k_{B} T}{\hbar \omega_{0}}\right)^{4}
$$

would be small even without the factor $\left(k_{B} T / \hbar \omega_{0}\right)^{4}$, which is already small by assumption.

At high temperatures all we need is $\mathcal{P}_{c l} \simeq \lambda \omega_{0}^{2}\langle X \dot{\xi}\rangle_{c l}$, with the expectation value from (6.17).

\subsection{Temperature-Dependence}

When both temperatures are low, which is the most likely scenario in practice, the prescription $\Delta_{T}$ cancels the component $\mathcal{P}^{(0)}$, whence

$$
\frac{k_{B} T_{1,2}}{\hbar \omega_{0}} \ll 1: \quad \mathcal{P} \simeq \mathcal{P}^{(1)} \simeq \frac{\lambda^{2} \hbar g^{2} \omega_{0}^{2}}{\left(1-\lambda^{2}\right)^{2}} \frac{2 \pi^{3}}{15}\left\{\left(\frac{k_{B} T_{1}}{\hbar \omega_{0}}\right)^{4}-\left(\frac{k_{B} T_{2}}{\hbar \omega_{0}}\right)^{4}\right\} .
$$

By coincidence, the temperature-dependence is the same as at long distances, where heatflows are dominated not by vdW forces but by photon exchange. In the denominator, LTE would replace $\left(1-\lambda^{2}\right)^{2} \rightarrow 1$.

When both temperatures are high, $\mathcal{P}$ is governed by (7.14):

$$
\frac{k_{B} T_{1,2}}{\hbar \omega_{0}} \gg 1: \quad \mathcal{P} \simeq \mathcal{P}_{c l} \simeq \frac{g \lambda^{2} \omega_{0}^{2}}{\left(\lambda^{2}+g^{2}\right)}\left\{\frac{k_{B}\left(T_{1}-T_{2}\right)}{2 \omega_{0}}+\frac{\hbar^{2} \omega_{0}}{24 \pi}\left(\frac{1}{k_{B} T_{1}}-\frac{1}{k_{B} T_{2}}\right)\right\} .
$$

When $T_{1}$ is high and $T_{2}$ is low, $\mathcal{P}$ combines the $T_{1}$ component of (8.13) with (8.9); and not with the $T_{2}$ component of (8.12), which features there only because, with both temperatures low, their separately dominant absolute-zero components cancel each other out. Thus

$$
\left(\frac{k_{B} T_{1}}{\hbar \omega_{0}} \gg 1, \frac{k_{B} T_{2}}{\hbar \omega_{0}} \ll 1\right): \quad \mathcal{P} \simeq \frac{\lambda^{2} g \omega_{0}^{2}}{\left(\lambda^{2}+g^{2}\right)}\left\{\left[\frac{k_{B} T_{1}}{2 \omega_{0}}+\frac{\hbar^{2} \omega_{0}}{24 \pi} \frac{1}{k_{B} T_{1}}\right]-\left[\frac{\hbar}{4}\right]\right\} .
$$

This is dominated by first term, roughly speaking as if the high-temperature oscillator were emitting (van-der-Waals-wise) but receiving no response. Note that the third (the low-temperature) correction outweighs the second (which stems from the high temperature contribution) :

$$
\text { (third term) } /(\text { second term })=6 \pi k_{B} T_{1} / \hbar \omega_{0} .
$$

In the denominators of both (8.13) and (8.14), LTE would replace $\left(\lambda^{2}+g^{2}\right) \rightarrow g^{2}$.

\subsection{Distance-Dependence}

Recall that, by assumption, $g \ll 1$, and that in atomic units $\lambda \sim 1 / r^{3}$.

If $T_{1}$ and $T_{2}$ are both low, then by (8.12)

$$
\mathcal{P} \propto \lambda^{2} g^{2} \sim g^{2} / r^{6} .
$$

If $T_{1}$ is high and $T_{2}$ low, or if both are high, then by (8.13) or (8.14)

$$
\mathcal{P} \propto \frac{\lambda^{2} g}{\left(\lambda^{2}+g^{2}\right)}
$$


Then if $\lambda^{2} \ll g^{2}$, i.e. at extremely long distances,

$$
r^{3} \gg 1 / g \gg 1: \quad \mathcal{P} \propto \lambda^{2} / g \sim 1 / g r^{6} .
$$

By contrast, if $\lambda^{2} \gg g^{2}$, i.e. at distances from short to long but not extremely long,

$$
r^{3} \gg 1 / g \gg 1: \quad \mathcal{P} \text { is proportional to } g \text { and independent of } r,
$$

so that the heat flow is governed wholly by the dissipation. By (8.5), this is likely to be the case when the dissipation is radiative. On the other hand, we have seen that at very small $r$ the dipole-dipole interaction needs amendment.

Acknowledgements It is a pleasure to acknowledge advice from Tom Philbin and Carsten Henkel, and to thank two anonymous referees for several very apposite comments.

Open Access This article is distributed under the terms of the Creative Commons Attribution 4.0 International License (http://creativecommons.org/licenses/by/4.0/), which permits unrestricted use, distribution, and reproduction in any medium, provided you give appropriate credit to the original author(s) and the source, provide a link to the Creative Commons license, and indicate if changes were made.

\section{Appendix 1: The Denominator $D^{2}(u, g)$}

At several points one needs the function $1 / D^{2}$, with $D^{2}$ defined in (3.7). Recall that its arguments $u \equiv \omega / \omega_{0}$ and $\quad g \equiv \eta / \omega_{0}$ are dimensionless. From

$$
D^{2}(u, g) \equiv u^{4}-\left(2-g^{2}\right) u^{2}+1=\left(1-u^{2}\right)^{2}+g^{2} u^{2}
$$

we see that

$$
\begin{gathered}
D^{2}=\left(1-u^{2}-i g u\right)\left(1-u^{2}+i g u\right), \\
D^{2}=\left[u^{2}-u_{+}^{2}\right]\left[u^{2}-u_{-}^{2}\right]=\left(u+u_{+}\right)\left(u-u_{+}\right)\left(u+u_{-}\right)\left(u-u_{-}\right), \\
u_{ \pm}^{2}=1-g^{2} / 2 \mp i g \sqrt{1-g^{2} / 4} \Rightarrow u_{ \pm}=-i g / 2 \pm \sqrt{1-g^{2} / 4} .
\end{gathered}
$$

Written as partial fractions

$$
\begin{gathered}
\frac{u}{D^{2}}=\frac{1}{2\left(u_{+}^{2}-u_{-}^{2}\right)}\left\{\frac{1}{u+u_{+}}+\frac{1}{u-u_{+}}-\frac{1}{u+u_{-}}-\frac{1}{u-u_{-}}\right\}, \\
=\frac{u}{\left(u_{+}^{2}-u_{-}^{2}\right)}\left\{\frac{1}{u^{2}-u_{+}^{2}}-\frac{1}{u^{2}-u_{-}^{2}}\right\}, \quad\left(u_{+}^{2}-u_{-}^{2}\right)=-2 i g \sqrt{1-g^{2} / 4} .
\end{gathered}
$$

Cauchy's theorem immediately yields the two remarkable the integrals

$$
\int_{-\infty}^{\infty} d u \frac{(g / \pi)}{D^{2}(u, g)}=\int_{-\infty}^{\infty} d u \frac{(g / \pi) u^{2}}{D^{2}(u, g)}=1 .
$$

That they are independent of $g$ illustrates the fact that, distributionally,

$$
\lim _{g \rightarrow 0} \frac{(g / \pi)}{D^{2}(u, g)}=\delta\left(u^{2}-1\right)=\frac{1}{2}[\delta(u-1)+\delta(u+1)] .
$$


We shall need also a related but divergent integral which, in an obvious shorthand, we write as

$$
\begin{aligned}
\lim _{\bar{u} \rightarrow \infty} \int_{-\bar{u}}^{\bar{u}} d u \frac{(g / \pi) u^{4}}{D^{2}(u, g)} & =\lim _{\bar{u} \rightarrow \infty} \int_{-\bar{u}}^{\bar{u}} d u \frac{g}{\pi}\left\{1-\frac{1}{D^{2}(u, g)}+\frac{\left(2-g^{2}\right) u^{2}}{D^{2}(u, g)}\right\} \\
& =\frac{2 g}{\pi} \bar{u}-1+\left(2-g^{2}\right)=\frac{2 g}{\pi} \bar{u}+\left(1-g^{2}\right) .
\end{aligned}
$$

\section{Appendix 2: Free Field on a Half-Line}

Recall the Fourier inversion formulae appropriate to our Neumann boundary conditions:

$$
\begin{gathered}
\int_{-\infty}^{0} d x \cos (\omega x) \cos \left(\omega^{\prime} x\right)=\int_{-\infty}^{0} d x \sin (\omega x) \sin \left(\omega^{\prime} x\right)=\frac{\pi}{2} \delta\left(\omega-\omega^{\prime}\right), \\
s(x)=\int_{0}^{\infty} d \omega \tilde{s}(\omega) \cos (\omega x), \quad \tilde{s}(\omega)=\frac{2}{\pi} \int_{-\infty}^{0} d x s(x) \cos (\omega x) .
\end{gathered}
$$

Correspondingly, for free fields, i.e. given $\mu=0$, the commutation rule (2.8)b can be satisfied by expanding

$$
\begin{gathered}
\phi(x, t)=\int_{0}^{\infty} d \omega \sqrt{\hbar / \pi \omega} \cos (\omega x)\left\{a(\omega) \exp (-i \omega t)+a^{+}(\omega) \exp (i \omega t)\right\}, \\
\Pi(x, t)=\dot{\phi}(x, t)=\int_{0}^{\infty} d \omega \sqrt{\hbar \omega / \pi} \cos (\omega x) i\left\{-a(\omega) \exp (-i \omega t)+a^{+}(\omega) \exp (i \omega t)\right\}, \\
{\left[a(\omega), a^{+}\left(\omega^{\prime}\right)\right]=\delta\left(\omega-\omega^{\prime}\right), \quad\left[a(\omega), a\left(\omega^{\prime}\right)\right]=0 .}
\end{gathered}
$$

Further, we define

$$
\begin{gathered}
Q(\omega)=\sqrt{\hbar / 2 \omega}\left[a^{+}(\omega)+a(\omega)\right], \quad P(\omega)=i \sqrt{\hbar \omega / 2}\left[a^{+}(\omega)-a(\omega)\right], \\
{\left[Q(\omega), P\left(\omega^{\prime}\right)\right]=i \hbar \delta\left(\omega-\omega^{\prime}\right),} \\
a(\omega)=\sqrt{\omega / 2 \hbar} Q(\omega)+i \sqrt{1 / 2 \hbar \omega} P(\omega), \quad a^{+}(\omega)=\sqrt{\omega / 2 \hbar} Q(\omega)-i \sqrt{1 / 2 \hbar \omega} P(\omega) .
\end{gathered}
$$

Then

$$
\begin{gathered}
\phi(x, t)=\int_{0}^{\infty} d \omega \sqrt{\frac{2}{\pi}} \cos (\omega x)\left\{Q(\omega) \cos (\omega t)+\frac{1}{\omega} P(\omega) \sin (\omega t)\right\} \\
\Pi(x, t)=\int_{0}^{\infty} d \omega \sqrt{\frac{2}{\pi}} \cos (\omega x)\{-\omega Q(\omega) \sin (\omega t)+P(\omega) \cos (\omega t)\} \\
\mathcal{H}_{\text {rad }} \equiv \int_{-\infty}^{0} d x \frac{1}{2}\left\{\Pi^{2}+\left(\frac{\partial \phi}{\partial x}\right)^{2}\right\} \\
=\int_{0}^{\infty} d \omega \frac{1}{2}\left\{P^{2}(\omega)+\omega^{2} Q^{2}(\omega)\right\}=\int_{0}^{\infty} d \omega \frac{1}{2} \hbar \omega\left\{a^{+}(\omega) a(\omega)+a(\omega) a^{+}(\omega)\right\}
\end{gathered}
$$




\section{Appendix 3: The Force Correlator $F(\tau)$}

To explore the correlator as a function of time, we use (4.21) to write it as

$$
\begin{aligned}
F(\tau) & =\frac{\eta \hbar}{2 \pi} \int_{-\infty}^{\infty} d \omega \omega \operatorname{coth}\left(\frac{\beta \hbar \omega}{2}\right) \exp (i \omega \tau) \\
& =\frac{2 \eta}{\pi \beta^{2} \hbar} \int_{-\infty}^{\infty} d x x \operatorname{coth}(x) \exp (i \Theta x), \quad \Theta \equiv \frac{2 \tau}{\beta \hbar},
\end{aligned}
$$

and exploit the expansions (4.23). The integral is a country cousin of those featuring in the autocorrelation functions of black-body radiation, found by Bourret [5] and detailed e.g. by Mehta and Wolf [20], [21].

To determine $F(\tau)$ exactly one substitutes into (11.1) from (4.23)b. Then one can close the contour in the upper half-plane, where the integrand has only the unit-residue Matsubara poles at $x_{n}=n \pi i$, with $n=1,2,3, \ldots$, and sum:

$$
\sum_{n=1}^{\infty} n \exp (-n \pi \Theta)=1 / 4 \sinh ^{2}(\pi \Theta / 2) \Rightarrow F(\tau)=-\frac{\pi \eta}{\beta^{2} \hbar} \frac{1}{\sinh ^{2}(\pi \tau / \beta \hbar)} .
$$

One gets the same result by identifying $\int_{0}^{\infty} d x x \cos (\Theta x)=-1 / \Theta^{2}$, integrating under $\sum_{n} \ldots$ term by term, and resumming. However, more interesting for present purposes than the exact expression are the asymptotics (i) in the high-temperature/classical regime, and (ii) in the low-temperature/essentially quantum regime.

(i) For nominally small $\hbar$ and/or high $T$, we expand coth $(\beta \hbar \omega / 2)$ in (11.1)a according to (4.23)a. This leads straightforwardly to

$$
F \simeq F_{c l}(\tau) \equiv \eta\left\{\frac{2}{\beta} \delta(\tau)-\frac{\beta \hbar^{2}}{6} \delta^{(2)}(\tau)-\frac{\beta^{3} \hbar^{4}}{360} \delta^{4}(\tau)+\cdots\right\},
$$

where we have used

$$
\int_{-\infty}^{\infty} d \omega \omega^{n} \exp (i \omega \tau)=2 \pi(-i)^{n} \delta^{(n)}(\tau), \quad \delta^{(n)}(\tau) \equiv \partial^{n} \delta(\tau) / \partial \tau^{n} .
$$

On the practical level, much labour can be saved on the way to (5.12) for $\left\langle q^{2}\right\rangle_{c l}$ and to (7.14) for $\langle X \dot{\xi}\rangle_{c l}$ by substituting (11.3) into (5.6) and (7.4) respectively.

(ii) By contrast, nominally large $\hbar$ and/or low $T$ suggest approximating the exact expression (11.2)b not via its Fourier transform, but by its Laurent series

$$
1 / \sinh ^{2}(z \rightarrow 0)=1 / z^{2}-1 / 3+z^{2} / 15-\ldots,
$$

leading to

$$
F \simeq F_{0} \equiv \eta\left\{-\frac{\hbar}{\pi \tau^{2}}+\frac{\pi}{3 \hbar \beta^{2}}-\frac{\pi^{3}}{15 \beta^{4} \hbar^{3}} \tau^{2}\right\}
$$

Unfortunately the first term makes the pertinent time integrations too awkward to be useful in practice. 


\section{Appendix 4: Half-Spaces}

\section{Generalities}

Our results on oscillators can be used to cast some light on the heat flux $\mathcal{R}$ between two identical Drude-modelled half-spaces a distance $Z$ apart, with dielectric functions

$$
\varepsilon(\omega)=1+\omega_{p}^{2} /\left[\omega_{0}^{2}-\omega^{2}-i \omega \Gamma\right], \quad \varepsilon(0)=1+\omega_{p}^{2} / \omega_{0}^{2}, \quad \Gamma=\omega_{p}^{2} / 4 \pi \sigma,
$$

where $\sigma$ is the conductivity. For simplicity, though unrealistically, we disregard the temperature-dependence of $\Gamma$. Section 6 of [3] explains that one may treat the system as consisting of pairs of coupled surface oscillators, each pair with common surface-parallel wave-vector $\mathbf{k}$, frequency $\omega_{S}$, damping constant $\Gamma$, and mutual coupling strength $\lambda$, where

$$
\omega_{S}^{2}=\omega_{0}^{2}+\omega_{p}^{2} / 2, \quad \beta_{S}^{2} \equiv \omega_{p}^{2} / 2 \omega_{S}^{2}=\frac{\varepsilon(0)-1}{\varepsilon(0)+1}, \quad 0 \leq \beta_{S}^{2} \leq 1, \quad \lambda=\beta_{S}^{2} \exp (-k Z) .
$$

Evidently

$$
\mathcal{R}=\int \frac{d^{2} k}{(2 \pi)^{2}} \mathcal{P}\left(\omega_{S}, \lambda\right),
$$

where $\mathcal{P}\left(\omega_{S}, \lambda\right)$ is given by (6.15) with $\omega_{0} \rightarrow \omega_{S}, \lambda$ from (12.2), and $g \rightarrow \Gamma / \omega_{S}$. Insulators have $\omega_{0} \neq 0$, hence $\varepsilon(0)>1$ but finite, and $\beta_{S}^{2}<1$. Conductors have $\omega_{0}=0$, hence $\varepsilon(0) \rightarrow \infty$, and $\beta_{S}^{2}=1$. Until further comment, our equations apply to both.

It must be stressed from the outset that this appendix is meant to exhibit only a few immediate and easy-to-find consequences of the basic results derived in the text for our prototype problem of a single pair of oscillators. It is outside the remit of the present paper, and outside the expertise of the present writer, to try and look for any more sophisticated implications that these basic results might have regarding steady-state heat flows or forces between other types or configurations of materials. (A few recent references to discussions of such systems were indicated at the end of Sect. 6.)

\section{High Temperatures}

The classical (high-temperature) limit is determined in Sect. 6 of [3], whose $\{\mathcal{P}, P, \beta, \bar{\gamma}, \zeta\}$ translate into our present $\left\{\mathcal{R}, \mathcal{P}, \beta_{S}, g, Z\right\}$. Equations $(6.5,6.6)$ there can be rearranged as

$$
\begin{gathered}
\mathcal{R}_{c l}=\frac{k_{B} \Delta T}{16 \pi Z^{2}} \beta_{S}^{2} \omega_{S} \mathcal{J}_{c l}(\Lambda), \\
\mathcal{J}_{c l} \equiv \frac{1}{\Lambda}\left\{\operatorname{dilog}\left[1+\left(\frac{1}{\Lambda^{2}}\right)\right]+2 \log ^{2}\left(\frac{1}{\Lambda}\right)+\frac{\pi^{2}}{6}\right\}, \quad \Lambda \equiv \frac{\beta_{S}^{2} \omega_{S}}{\Gamma} .
\end{gathered}
$$

This agrees with the classical limit of the general expression derived by Loomis and Maris [19], who do not assume LTE, but only that in each half-space the noise is appropriate to the temperature of its thermostat. Asymptotically

$$
\begin{gathered}
\mathcal{J}_{c l}(\Lambda \ll 1)=\Lambda-\frac{\Lambda^{3}}{4}+\frac{\Lambda^{5}}{9}+\cdots \\
\mathcal{J}_{c l}(\Lambda \gg 1)=\frac{1}{\Lambda}\left\{\left[\frac{\pi^{2}}{6}+2 \log ^{2}(\Lambda)\right]-\frac{1}{\Lambda^{3}}+\frac{1}{4 \Lambda^{5}}+\cdots\right\},
\end{gathered}
$$


with a single (rather flat) maximum

$$
\mathcal{J}_{c l, \max } \simeq 1.36, \text { at } \Lambda_{\max } \simeq 4.48 .
$$

Accordingly, regardless of $\Gamma$,

$$
\mathcal{R}_{c l} \leq\left(k_{B} \Delta T / 16 \pi Z^{2}\right) \beta_{S}^{2} \omega_{S} \mathcal{J}_{c l, \max }
$$

Re-focussing on the individual constituent oscillator-pairs with given $\mathbf{k}$, we see from (12.2)d that regardless of $Z$ and $\mathbf{k}$, and again regardless of $\Gamma$, classically

$$
\beta_{S}^{2} \ll 1 \Rightarrow \lambda \ll 1 \Rightarrow \text { weak coupling } \Rightarrow L T E .
$$

Conversely, (6.19) for $\mathcal{P}_{L T E, c l}$, adapted by the rules given just below (12.3), leads to

$$
\begin{aligned}
\mathcal{R}_{L T E, c l} & =\int \frac{d^{2} k}{(2 \pi)^{2}} \mathcal{P}_{L T E, c l}\left(\omega_{S}, \lambda\right) \\
& =\frac{\beta_{S}^{4} \omega_{S}^{2} k_{B} \Delta T}{4 \pi \Gamma} \int_{0}^{\infty} d k k \exp (-2 k Z)=\left(\frac{k_{B} \Delta T}{16 \pi Z^{2}}\right) \beta_{S}^{2} \omega_{S} \Lambda,
\end{aligned}
$$

which should be compared with (12.9).

Without $L T E$, approximations to $\mathcal{J}_{c l}$ do, via $\Lambda$, depend strongly on $\Gamma$. By $(12.6,12.7$, 12.11)

$$
\mathcal{R}_{c l}(\Lambda \ll 1) \simeq \frac{k_{B} \Delta T}{16 \pi Z^{2}} \frac{\omega_{S}^{2} \beta_{S}^{4}}{\Gamma}=\mathcal{R}_{L T E, c l}, \quad \mathcal{R}_{c l}(\Lambda \gg 1) \simeq \frac{k_{B} \Delta T}{8 \pi Z^{2}} \Gamma \log ^{2}\left(\frac{\beta_{S}^{2} \omega_{S}}{\Gamma}\right) .
$$

Classically therefore $L T E$ fails unless $\Lambda$ is small.

Up to this point, and subject to the stated conditions, the conclusions in this appendix apply both to conductors and to insulators. But conductors have $\beta_{S}^{2}=1$, usually with $\omega_{S} / \Gamma \gg 1$. Then $\Lambda \gg 1$, whence (12.12)b applies, so that

$$
\text { conductors : } \quad \mathcal{R}_{c l} \simeq \frac{k_{B} \Delta T}{8 \pi Z^{2}} \Gamma \log ^{2}\left(\frac{\omega_{S}}{\Gamma}\right),
$$

and LTE fails automatically.

\section{Low Temperatures}

At low $T$, Eq. (12.2) plus (8.12) with $\omega_{0} \rightarrow \omega_{S}$ eventually lead to

$$
\begin{aligned}
\mathcal{R}_{0} & =\frac{\pi^{2} \hbar \Gamma^{2}}{60 Z^{2}} \Delta_{T}\left(\frac{k_{B} T}{\hbar \omega_{S}}\right)^{4} \beta_{S}^{4} \int_{0}^{\infty} d x \frac{\exp (-x)}{\left[1-\beta_{S}^{4} \exp (-x)\right]^{2}} \\
& =-\frac{\pi^{2} \hbar \Gamma^{2}}{60 Z^{2}} \Delta_{T}\left(\frac{k_{B} T}{\hbar \omega_{S}}\right)^{4} \log \left(1-\beta_{S}^{4}\right) .
\end{aligned}
$$

This can be applied to insulators, but not to conductors, for which $\omega_{0}=0 \Rightarrow \beta_{S}=1$. The basic physical reason is that $\omega_{0}=0$ is incompatible with our underlying low- $T$ approximation to oscillator pairs, which hinges on $k_{B} T / \hbar \omega_{0} \ll 1$. Unfortunately this puts our expressions for $\mathcal{R}_{0}$ out of touch with the low-temperature measurements of Kralik et al. [17] on tungsten. 
Finally, leading-order perturbation theory, and thereby LTE, mean leading order in $\beta_{S}^{2}$, replacing $-\log \left(1-\beta_{S}^{4}\right) \rightarrow \beta_{S}^{4}$, whence

$$
L T E \Leftrightarrow \beta_{S}^{2} \ll 1 \Rightarrow R_{L T E, 0} \simeq \frac{4 \pi^{2} \hbar \Gamma^{2}}{15 Z^{2}} \Delta_{T}\left(\frac{k_{B} T}{\hbar \omega_{p}}\right)^{4}=\frac{1}{60 Z^{2}} \frac{\Delta_{T}\left(k_{B} T\right)^{4}}{\hbar^{3} \sigma^{2}} .
$$

\section{References}

1. Barnett, S.M., Cresser, J.D., Croke, S.: Theory of the strongly-damped quantum harmonic oscillator. arXiv:1508.02442 (2015)

2. Barton, G.: Elements of Green's Functions and Propagation. Oxford University Press, Oxford (1989)

3. Barton, G.: Classical van der Waals heat flow between oscillators and between half-spaces. J Phys Condens Matter 27, 214005 (2015)

4. Bimonte, G.: Observing the Casimir-Lifshitz force out of thermal equilibrium. Phys Rev A 92, 032116 (2015)

5. Bourret, R.C.: Coherence properties of blackbody radiation. Nuovo Cimento 18, 345 (1960)

6. Caldeira, A.O., Leggett, A.J.: Path integral approach to quantum Brownian motion. Physica 121A, 587 (1983)

7. Caldeira, A.O., Leggett, A.J.: Quantum tunnelling in a dissipative system. Ann. Phys. (N.Y.) 149, 374 (1983)

8. Ciliberto, S., Imparato, A., Naert, A., Tanase, M.: Heat transfer between two nanoparticles through near field interaction. Phys. Rev. Lett. 110, 180601 (2013)

9. Ciliberto, S., Imparato, A., Naert, A., Tanase, M.: Statistical properties of the energy exchanged between two heat baths coupled by thermal fluctuations. J. Stat. Mech. 2013, 12014 (2013)

10. Domingues, G., Volz, S., Joulain, K., Greffet, J.-J.: Heat transfer between two nanoparticles through near field interactions. Phys. Rev. Lett. 94, 085901 (2005)

11. Dorofeyev, I.: Coupled quantum oscillators within independent quantum reservoirs. Can. J. Phys. 91, 537 (2013)

12. Dubi, Y., Di Ventra, M.: Heat flow and thermoelectricity in atomic and molecular junctions. Rev. Mod. Phys. 83, 131 (2011)

13. Ghesquière, A., Sinayskiy, I., Petruccione, F.: Non-equilibrium thermal entanglement in a two-particle system. Phys. Scr. T151, 014017 (2012)

14. Ghesquière, A., Sinayskiy, I., Petruccione, F.: Dynamics and nonequilibrium steady state in a system of coupled harmonic oscillators. Phys. Lett. A 377, 1682 (2013)

15. Grabert, H., Weiss, U., Talkner, P.: Quantum theory of the damped harmonic oscillator. Z. Phys. B 55, 87 (1984)

16. Intravaia, F., Behunin, R.O., Henkel, C., Bush, K., Dalvit, D.A.R.: Failure of local thermal equilibrium in quantum friction. Phys. Rev. Lett. 17, 100402 (2016)

17. Kralik, T., et al.: Strong near-field enhancement of radiative heat transfer between metallic surfaces. Phys. Rev. Lett. 109, 224302 (2012)

18. Krüger, M., Bimonte, G., Emig, T., Kardar, M.: Near-equilibrium electromagnetic fluctuations: heat transfer and interactions. Phys. Rev. B 86, 115423 (2012)

19. Loomis, J.L., Maris, H.J.: Theory of heat transfer by evanescent electromagnetic waves. Phys. Rev. B 50(18), 517 (1994)

20. Mehta, C.L., Wolf, E.: Coherence properties of blackbody radiation. I. Correlation tensors of the classical field. Phys. Rev. 134, A1143 (1946)

21. Mehta, C.L., Wolf, E.: Coherence properties of blackbody radiation. II. Correlation tensors of the quantized field. Phys. Rev. 134, A1149 (1946)

22. Messina, R., Antezza, M.: Scattering matrix approach to Casimir-Lifshitz force and heat transfer out of thermal equilibrium between arbitrary bodies. Phys. Rev. A 84, 042102 (2011)

23. Ottens, R.S., et al.: Near-field radiative heat transfer between macroscopic planar surfaces. Phys. Rev. Lett. 107, 014301 (2011)

24. Philbin, T.G., Anders, J.: Thermal energies of classical and quantum damped oscillators coupled to reservoirs. J. Phys. A 49, 21503 (2016)

25. Unruh, W.G., Zurek, W.H.: Reduction of a wave packet in quantum Brownian motion. Phys. Rev. D 40, 1071 (1989) 